\title{
Numerical methods for solving initial value problems of some kinds of nonlinear impulsive fractional differential equations
}

\author{
Yuanfeng Jin ${ }^{\mathrm{a}, *}$, Choehui Chol ${ }^{\mathrm{b}}$, Paksun $\mathrm{A} \mathrm{e}^{\mathrm{b}}$, Jongkum Song ${ }^{\mathrm{b}}$, Gang $\mathrm{Lu}^{\mathrm{c}}$ \\ ${ }^{a}$ Department of Mathematics, Yanbian University, Jilin 133002, China. \\ ${ }^{b}$ Department of Mathematics, Kim II-sung University, DPRK.
}

${ }^{c}$ Department of Mathematics, School of Science, Shenyang University of Technology, Shenyang 110178, China.

Communicated by K. Q. Lan

\begin{abstract}
This article is concerned with the numerical solutions for initial value problems of nonlinear impulsive fractional differential equations which are actively studied recently. In this paper we construct numerical schemes for solving initial value problems of I-type impulsive fractional differential equation and II-type impulsive fractional differential equation and estimate their convergence and stability.
\end{abstract}

Keywords: Caputo fractional derivative, impulsive fractional differential equation, difference method, operational matrix method, decomposition method.

2010 MSC: 65L05, 34A37, 34H05.

(C)2018 All rights reserved.

\section{Introduction}

Fractional differential equations have recently proved to be commonly used in different research areas such as engineering, physics, chemistry, economics, etc. Especially, they draw a great application in nonlinear oscillations of earthquakes, see page flow in porous media and in fluid dynamic traffic model, and many physical phenomena $[6,10,11,13,14,17,21]$.

Recently, Mahto et al. [12] investigated the existence and uniqueness of solutions of Caputo impulsive fractional differential equations with $0<\alpha<1$ as below.

$$
\begin{cases}{ }^{c} D^{\alpha} x(t)=f(t, x(t)), & t \in I=[0,1], t \neq t_{k}, \\ \left.\Delta x(t)\right|_{t=t_{k}}=I_{k}\left(x\left(t_{k}^{-}\right)\right), & k=1,2, \ldots, m \\ x(0)=0, & \end{cases}
$$

\footnotetext{
*Corresponding author

Email addresses: yfkim@ybu.edu.cn (Yuanfeng Jin), 1214181195@qq.com (Choehui Chol), 1060208723@qq.com (Paksun Ae), 1179667503@qq.com (Jongkum Song), lvgang1234@hanmail . net (Gang Lu)
}

doi: $10.22436 /$ jnsa.011.10.03

Received: 2016-01-12 Revised: 2017-11-18 Accepted: 2018-06-08 
Chang [5] studied the existence of solution for the impulsive fractional differential equations with integral boundary condition as follows.

$$
\begin{cases}{ }^{c} D^{\alpha} y(t)=f(t, y(t)), & t \in J=[0,1], t \neq t_{k}, \\ \left.\Delta y(t)\right|_{t=t_{k}}=I_{k}\left(y\left(t_{k}^{-}\right)\right), & k=\overline{1, m}, \\ y(0)=\int_{0}^{1} g(s) y(s) d s, & \end{cases}
$$

In a recent work [3], Belmekki et al. studied the existence of solution to the following periodic boundary value problem for a nonlinear impulsive fractional differential equation by Schaeffer's fixed point theorem.

$$
\left\{\begin{array}{l}
D_{t_{k}^{+}}^{\alpha} \mu(t)-\lambda \mu(t)=f(t, \mu(t)), t \in\left(t_{k}, t_{k+1}\right), k=0,1, \ldots, p, \\
\lim _{t \rightarrow t_{k}^{+}}\left(t-t_{k}\right)^{1-\delta}\left(\mu(t)-\mu\left(t_{k}\right)\right)=I_{k}\left(\mu\left(t_{k}\right)\right), k=1,2, \ldots, p, \\
\lim _{t \rightarrow 0_{+}} t^{1-\delta} \mu(t)=\mu(1) .
\end{array}\right.
$$

And they $[1,2,4,7,22,23]$ also investigated the existence and uniqueness of solutions for impulsive fractional differential equations.

While almost papers about the impulsive fractional differential equations were written in respect to the existence and uniqueness of solutions, few papers treated the numerical method for solving impulsive fractional differential equations.

Randelovic et al. [18] studied a difference approximation algorithm for the integer order impulsive differential equation with initial condition and gave an example, but they did not investigate the numerical analysis for their method.

Yang et al. [24] proposed an effective numerical method for time-space fractional Fokker-Planck equation and proved the stability and convergence of their method, but it is easy to see that the constants of stability and convergence grow as the iteration number increases.

$$
\begin{aligned}
\frac{\partial^{\alpha} \mu(x, t)}{\partial t^{\alpha}} & =\left[\frac{\partial}{\partial x} \frac{V^{\prime}(x)}{m \eta_{\alpha}}+K_{\alpha}^{\mu} \frac{\partial^{\mu}}{\partial|x|^{\mu}}\right] \mu(x, t)+S(\mu(x, t), x, t), \\
\mu(a, t) & =\mu(b, t)=0,0 \leqslant t \leqslant T, \\
\mu(x, 0) & =\mu_{0}(x), a<x<b .
\end{aligned}
$$

Hariharan et al. [9] showed broadly that Haar wavelet method is a very effective and powerful tool in solving linear and nonlinear differential equations. Habibollah saeedi et al. [20]reduced the fractional Volterra and Abel integral equations to a system of algebraic equations, estimated a global error bound and gave some numerical examples by using Haar wavelet operational matrix. Neamaty et al. [15] proposed a solving method for fractional partial differential equation with Caputo fractional derivative by using Haar wavelet operational matrix and gave a numerical example.

Motivated by the above researches we firstly study the effective difference scheme for the following impulsive fractional differential equation (II-type impulsive fractional differential equation) and estimated the stability and convergence of our scheme

$$
\left\{\begin{array}{l}
{ }^{c} D_{t_{k}}^{\alpha} x(t)=f(t, x(t)), t \in\left(t_{k}, t_{k+1}\right), k=0,1, \ldots, m, \\
\left.\Delta x(t)\right|_{t=t_{k}}=I_{k}\left(\mu\left(t_{k}^{-}\right)\right), k=1,2, \ldots, m, \\
x(0)=0 .
\end{array}\right.
$$

And we propose the wavelet operational matrix method for the other kind of impulsive fractional differential equation (I-type impulsive fractional differential equation), analyze the convergence of our method, and give an numerical example.

$$
\left\{\begin{array}{l}
{ }^{c} D_{0}^{\alpha} x(t)=f(t, x(t)), t \in(0,1], t \neq t_{k} \in(0,1) \\
\left.\Delta x(t)\right|_{t=t_{k}}=I_{k}\left(\mu\left(t_{k}^{-}\right)\right), k=1,2, \ldots, l \\
x(0)=x_{0}
\end{array}\right.
$$


In above two equations, we assume that

$$
f \in C(I \times X, X), \quad I_{k}: X \rightarrow X, \quad 0<\alpha<1,
$$

and put as follows.

$$
\begin{aligned}
0 & =t_{0}<t_{1}<\cdots<t_{m}<t_{m+1}=1, \\
\left.\Delta x(t)\right|_{t=t_{k}} & =x\left(T_{k}^{+}\right)-x\left(t_{k}^{-}\right), \\
x\left(t_{k}^{+}\right) & :=\lim _{h \rightarrow+0} x\left(t_{k}+h\right), x\left(t_{k}^{-}\right):=\lim _{h \rightarrow+0} x\left(t_{k}-h\right) .
\end{aligned}
$$

\section{II-type impulsive fractional differential equation and the effective difference scheme}

2.1. II-type impulsive fractional differential equation

Definition 2.1. The equation (2.1) is said to be an II-type impulsive fractional differential equation.

$$
\left\{\begin{array}{l}
{ }^{c} D_{t_{k}}^{\alpha} x(t)=f(t, x(t)), t \in\left(t_{k}, t_{k+1}\right), k=0,1, \ldots, m \\
\left.\Delta x(t)\right|_{t=t_{k}}=I_{k}\left(x\left(t_{k}^{-}\right)\right), k=1,2, \ldots, m \\
x(0)=0
\end{array}\right.
$$

where

$$
\begin{aligned}
f & \in C(I \times X, X), \\
I_{k}: X & \rightarrow X, \\
0 & =t_{0}<t_{1}<\cdots<t_{m}<t_{m+1}=1, \\
\left.\Delta x(t)\right|_{t=t_{k}} & =x\left(t_{k}^{+}\right)-x\left(t_{k}^{-}\right), \\
x\left(t_{k}^{+}\right) & =\lim _{h \rightarrow+0} x\left(t_{k}+h\right), x\left(t_{k}^{-}\right)=\lim _{h \rightarrow+0} x\left(t_{k}-h\right) .
\end{aligned}
$$

In this section, we assume that $1 / 2<\alpha<1$.

\subsection{The effective difference scheme}

Denote as follows:

$$
\mathrm{T}_{\mathrm{k}}:=\mathrm{t}_{\mathrm{k}+1}-\mathrm{t}_{\mathrm{k}}, \quad \tau_{\mathrm{k}}:=\mathrm{T}_{\mathrm{k}} / \mathrm{N}_{\mathrm{k}}, \quad \mathrm{t}_{\mathrm{n}}^{\mathrm{k}}:=\mathrm{t}_{\mathrm{k}}+\mathrm{n} \tau_{\mathrm{k}}, \quad \mathrm{N}_{\mathrm{k}} \in \mathrm{N}, \mathrm{n}=\overline{0 . \mathrm{N}_{\mathrm{k}}}, \mathrm{k}=\overline{0, \mathrm{~m}} .
$$

Firstly, adopting the L1-algorithm [16], we discretize the Caputo time fractional derivative as

$$
{ }^{c} D^{\alpha} x\left(t_{n+1}\right)=\frac{\tau^{-\alpha}}{\Gamma(2-\alpha)} \sum_{j=0}^{n} b_{j}\left(x\left(t_{n+1-j}\right)-x\left(t_{n-j}\right)\right)+O\left(\tau^{1+\tau}\right),
$$

where $\tau=T / N, t_{n}=n \tau, n=\overline{0, N}$ and $b_{j}=(j+1)^{1-\alpha}-j^{1-\alpha}$. The nonlinear function $f$ can be discretized as

$$
f\left(t_{n+1}^{k}, x\left(t_{n+1}^{k}\right)\right)=f\left(t_{n}^{k}, x\left(t_{n}^{k}\right)\right)+O\left(\tau_{k}\right) .
$$

Therefore, using (2.2)-(2.3), we have

$$
\frac{\tau_{k}^{-\alpha}}{\Gamma(2-\alpha)} \sum_{j=0}^{n} b_{j}\left(x\left(t_{n+1-j}^{k}\right)-x\left(t_{n-j}^{k}\right)\right)=f\left(t_{n}^{k}, x\left(t_{n}^{k}\right)\right)+O\left(\tau_{k}\right) .
$$


Put $\mu_{k}:=\tau_{k}^{\alpha} \Gamma(2-\alpha)$. From (2.4) we can get

$$
x\left(t_{n+1}^{k}\right)=b_{n} x\left(t_{0}^{k}\right)+\sum_{j=0}^{n-1}\left(b_{j}-b_{j+1}\right) x\left(t_{n-j}^{k}\right)+\mu_{k} f\left(t_{n}^{k}, x\left(t_{n}^{k}\right)\right)+R_{n+1}^{k},
$$

where there exists a positive constant $c_{1}$ such that $\left|R_{n+1}^{k}\right| \leqslant c_{1} \tau_{k}^{1+\alpha}$. Let $x_{n}^{k}$ be the numerical approximation of $x\left(t_{\mathfrak{n}}^{k}\right)$, and $f_{\mathfrak{n}}^{k}$ the numerical approximation of $f\left(t_{\mathfrak{n}}^{k}, x\left(t_{\mathfrak{n}}^{k}\right)\right)$. Then we obtain the following effective difference scheme for the first equation of problem (2.1):

$$
x_{n+1}^{k}=b_{n} x_{0}^{k}+\sum_{j=0}^{n-1}\left(b_{j}-b_{j+1}\right) x_{n-j}^{k}+\mu_{k} f_{n}^{k}, \quad x_{1}^{k}=b_{0} x_{0}^{k}+\mu_{k} f_{0}^{k}, \quad n=\overline{1, N_{k}-1}, k=\overline{0, m} .
$$

The second expression of problem (2.1) can be written as follows:

$$
x\left(t_{k+1}+0\right)=x\left(t_{k+1}-0\right)+I_{k+1}\left(x\left(t_{k+1}-0\right)\right), k=\overline{0, m} .
$$

Thus, we obtain the approximation

$$
x_{0}^{k}=x_{N_{k-1}}^{k-1}+I_{k}\left(x_{N_{k-1}}^{k-1}\right), k=1,2, \ldots, m, \quad x_{0}^{0}=x_{0} .
$$

Finally, we can get the effective difference scheme (2.6)-(2.7).

In the sequel, we assume that the functions $f$ and $I_{k}$ are Lipschitz continuous, that is,

$$
\begin{gathered}
\exists L>0 ; \forall x, y \in R,|f(t, x)-f(t, y)| \leqslant L|x-y|, \\
\exists L_{k}>0 ; \forall x, y \in R,\left|I_{k}(x)-I_{k}(y)\right| \leqslant L_{k}|x-y| .
\end{gathered}
$$

\subsection{Stability of the effective difference scheme}

Let $x_{n}^{k}$ and $\widetilde{x}_{n}^{k}$ be the approximate solutions of (2.6)-(2.7) with different initial conditions $x_{0}^{0}=x_{0}$ and $\widetilde{x}_{0}^{0}=x_{0}+\delta$.

Denote as follows:

$$
\rho_{n}^{k}:=x_{n}^{k}-\widetilde{x}_{n}^{k}, \quad f_{n}^{k}:=f\left(t_{n}^{k}, x_{n}^{k}\right), \quad \tilde{f}_{n}^{k}:=f\left(t_{n}^{k}, \tilde{x}_{n}^{k}\right) .
$$

Definition 2.2. The effective difference scheme (2.6)-(2.7) is said to be stable if

$$
\exists c_{0}>0 ; \max _{n, k}\left|\rho_{n}^{k}\right| \leqslant c_{0}\left|\rho_{0}^{0}\right| \text {. }
$$

Lemma 2.3. The effective difference scheme (2.6)-(2.7) is stable if

$$
\begin{aligned}
\tau_{k}^{\alpha} & \leqslant \frac{1}{\Gamma(2-\alpha) L} \min \left\{\sum_{j=1}^{n} b_{j} e^{-\left(n \tau_{k}\right)^{1-\alpha}}\left(e^{\left((n-j+1) \tau_{k}\right)^{1-\alpha}}-e^{\left((n-j) \tau_{k}\right)^{1-\alpha}}\right),\left(e^{\tau_{k}^{1-\alpha}}-1\right)\right\}, \\
n & =\overline{1, N_{k}}, k=\overline{0, m} .
\end{aligned}
$$

Proof. Put $\beta:=1-\alpha$ and $\left\|\rho_{\mathfrak{n}}^{k}\right\|_{n, k}:=e^{-\left(n \tau_{k}\right)^{\beta}}\left|\rho_{n}^{k}\right|$. It is obvious that

$$
e^{-1}\left|\rho_{n}^{k}\right| \leqslant\left\|\rho_{n}^{k}\right\|_{n, k}
$$

holds. 
i) The case of $k=0$.

At first we can get $\left\|\rho_{0}^{0}\right\|_{0,0}=|\delta|$, when $n=0$. For $n=1$, after some manipulation, we obtain

$$
\left|\rho_{1}^{0}\right| \leqslant b_{0}\left|\rho_{0}^{0}\right|+\mu_{0} L\left|\rho_{0}^{0}\right|, \quad\left\|\rho_{1}^{0}\right\|_{1,0} \leqslant e^{-\tau_{0}^{\beta}}\left(b_{0}+\mu_{0} \mathrm{~L}\right)\left|\rho_{0}^{0}\right| .
$$

From (2.10) we have

$$
e^{-\tau_{0}^{\beta}}\left(b_{0}+\mu_{0} L\right) \leqslant e^{-\tau_{0}^{\beta}}\left(1+\Gamma(2-\alpha) \cdot \frac{1}{\Gamma(2-\alpha) L}\left(e^{-\tau_{0}^{1-\alpha}}-1\right) \cdot L\right)=1 .
$$

Thus we can see easily that

$$
\left\|\rho_{1}^{0}\right\|_{1,0} \leqslant\left\|\rho_{0}^{0}\right\|_{0,0}
$$

Now suppose that

$$
\left\|\rho_{n}^{0}\right\|_{n, 0} \leqslant\left\|\rho_{0}^{0}\right\|_{0,0}, n=2,3, \ldots, l,
$$

when $n=l+1$, from (2.6) we have

$$
\left|\rho_{l+1}^{0}\right| \leqslant b_{l}\left|\rho_{0}^{0}\right|+\sum_{j=0}^{l-1}\left(b_{j}-b_{j+1}\right)\left|\rho_{l-j}^{0}\right|+\mu_{0} \mathrm{~L}\left|\rho_{l}^{0}\right| .
$$

Therefore, we obtain

$$
\begin{aligned}
& \left\|\rho_{l+1}^{0}\right\|_{l+1,0}=e^{-\left((l+1) \tau_{0}\right)^{\beta}}\left|\rho_{l+1}^{0}\right| \\
& \leqslant b_{l} e^{-\left((l+1) \tau_{0}\right)^{\beta}}\left|\rho_{0}^{0}\right|+\sum_{j=0}^{l-1}\left(b_{j}-b_{j+1}\right) e^{-\left((l+1) \tau_{0}\right)^{\beta}+\left((l-j) \tau_{0}\right)^{\beta}}\left\|\rho_{l-j}^{0}\right\|_{l-j, 0} \\
& +\mu_{0} \mathrm{~L} e^{-\left((l+1) \tau_{0}\right)^{\beta}+\left(l \tau_{0}\right)^{\beta}}\left\|\rho_{l}^{0}\right\|_{l, 0} \\
& \leqslant\left[b_{l} e^{-\left((l+1) \tau_{0}\right)^{\beta}}+\sum_{j=0}^{l-1}\left(b_{j}-b_{j+1}\right) e^{-\left((l+1) \tau_{0}\right)^{\beta}+\left((l-j) \tau_{0}\right)^{\beta}}+\mu_{0} L e^{-\left((l+1) \tau_{0}\right)^{\beta}+\left(l \tau_{0}\right)^{\beta}}\right]\left\|\rho_{0}^{0}\right\|_{0,0} \\
& =\left[b_{l} e^{-\left(l \tau_{0}\right)^{\beta}}+\sum_{j=1}^{l-1}\left(b_{j}-b_{j+1}\right) e^{-\left(l \tau_{0}\right)^{\beta}+\left((l-j) \tau_{0}\right)^{\beta}}=b_{0}-b_{1}+\mu_{0} L\right] e^{-\left((l+1) \tau_{0}\right)^{\beta}+\left(l \tau_{0}\right)^{\beta}}\left\|\rho_{0}^{0}\right\|_{0,0} \\
& \leqslant\left[b_{l} e^{-\left(l \tau_{0}\right)^{\beta}}+\sum_{j=1}^{l-1}\left(b_{j}-b_{j+1}\right) e^{-\left(l \tau_{0}\right)^{\beta}+\left((l-j) \tau_{0}\right)^{\beta}}-b_{1}+\mu_{0} L+1\right]\left\|\rho_{0}^{0}\right\|_{0,0} .
\end{aligned}
$$

Define $C:=b_{l} e^{-\left(l \tau_{0}\right)^{\beta}}+\sum j=1^{l-1}\left(b_{j}-b_{j+1}\right) e^{-\left(l \tau_{0}\right)^{\beta}+\left((l-j) \tau_{0}\right)^{\beta}}-b_{1}+\mu_{0} L$. Then we have

$$
\begin{aligned}
C & =b_{l} e^{-\left(l \tau_{0}\right)^{\beta}}+\sum_{j=1}^{l-1} b_{j} e^{-\left(l \tau_{0}\right)^{\beta}+\left((l-j) \tau_{0}\right)^{\beta}}-\sum_{j=1}^{l-1} b_{j+1} e^{-\left(l \tau_{0}\right)^{\beta}+\left((l-j) \tau_{0}\right)^{\beta}}-b_{1}+\mu_{0} L \\
& =\sum_{j=1}^{l} b_{j} e^{-\left(l \tau_{0}\right)^{\beta}+\left((l-j) \tau_{0}\right)^{\beta}}-\sum_{j=0}^{l-1} e^{-\left(l \tau_{0}\right)^{\beta}+\left((l-j) \tau_{0}\right)^{\beta}}+\mu_{0} L \\
& =\sum_{j=1}^{l} b_{j} e^{-\left(l \tau_{0}\right)^{\beta}+\left((l-j) \tau_{0}\right)^{\beta}}-\sum_{j=1}^{l} b_{j} e^{-\left(l \tau_{0}\right)^{\beta}+\left((l-j+1) \tau_{0}\right)^{\beta}}+\mu_{0} L \\
& =\sum_{j=1}^{l} b_{j} e^{-\left(l \tau_{0}\right)^{\beta}}\left(e^{\left((l-j) \tau_{0}\right)^{\beta}}-e^{\left((l-j+1) \tau_{0}\right)^{\beta}}\right)+\mu_{0} L .
\end{aligned}
$$


Using (2.10), we obtain

$$
C \leqslant-\Gamma(2-\alpha) L \tau_{0}^{\alpha}+\mu_{0} L=0
$$

Thus we get

$$
\left\|\rho_{l+1}^{0}\right\|_{l+1,0} \leqslant\left\|\rho_{0}^{0}\right\|_{0,0}
$$

Consequently, we can see that

$$
\left\|\rho_{n}^{0}\right\|_{n, 0} \leqslant\left\|\rho_{0}^{0}\right\|_{0,0}, n=\overline{1, N_{0}} .
$$

Substituting (2.11) into (2.12) shows that

$$
\left|\rho_{n}^{0}\right| \leqslant e\left\|\rho_{n}^{0}\right\|_{n, 0} \leqslant e\left\|\rho_{0}^{0}\right\|_{0,0}=e|\delta| .
$$

ii) The case of $k=1$.

From (2.7), we have

$$
x_{0}^{k}=x_{N_{k-1}}^{k-1}+I_{k}\left(x_{N_{k-1}}^{k-1}\right), \quad \tilde{x}_{0}^{k}=\widetilde{x}_{N_{k-1}}^{k-1}+I_{k}\left(\widetilde{x}_{N_{k-1}}^{k-1}\right), \quad k=\overline{1, m} .
$$

Thus we can see that

$$
\rho_{0}^{1}=\rho_{n_{0}}^{0}+I_{1}\left(\chi_{N_{0}}^{0}\right)-I_{1}\left(\widetilde{\chi}_{N_{0}}^{0}\right), \quad\left|\rho_{0}^{1}\right| \leqslant\left|\rho_{N_{0}}^{0}\right|+L_{1}\left|\rho_{N_{0}}^{0}\right| \leqslant\left(1+L_{1}\right)\left|\rho_{N_{0}}^{0}\right| \leqslant\left(1+L_{1}\right) e\left|\rho_{0}^{0}\right| .
$$

Similarly to i), we have

$$
\left|\rho_{n}^{1}\right| \leqslant\left(1+L_{1}\right) e^{2}\left|\rho_{0}^{0}\right| .
$$

iii) The case of $k \geqslant 2$.

For any $k \geqslant 2$, we can see easily that

$$
\left|\rho_{n}^{k}\right| \leqslant e^{k+1} \prod_{i=1}^{k}\left(1+L_{i}\right) \cdot\left|\rho_{0}^{0}\right| .
$$

The proof of Lemma 2.3 is completed.

Lemma 2.4 ([25]). The coefficients $b_{j}$ satisfy

(i)

$$
\forall j \in N, b_{j}>0
$$

(ii)

$$
1=b_{0}>b_{1}>\cdots>b_{n}, b_{n} \rightarrow 0 \text { as } n \rightarrow \infty ;
$$

(iii) when $0<\alpha<1$,

$$
\lim _{j \rightarrow \infty} \frac{b_{j}^{-1}}{j^{\alpha}}=\lim _{j \rightarrow \infty} \frac{j^{-1}}{\left(1+j^{-1}\right)^{1-\alpha}}=\frac{1}{1-\alpha} .
$$

Thus there is a positive constant $\mathrm{C}$ such that

$$
b_{j}^{-1} \leqslant C J^{\alpha}, j=0,1,2, \ldots
$$

Lemma 2.5. The coefficients $b_{j}$ satisfy 
(i) $\forall j \in N, b_{j} b_{j} \leqslant b_{j-1} b_{j+1}$;

(ii) $\min _{j \in\{1,2, \ldots, n\}}=\left\{\begin{array}{l}b_{n^{\prime}}^{2}, n=2 n^{\prime}, n^{\prime} \in N \\ b_{n^{\prime}} b_{n^{\prime}+1}, n=2 n^{\prime}+1, n^{\prime} \in N\end{array}\right.$

(iii) $b_{2}<2^{\alpha} b_{3}<3^{\alpha} b_{4}<\cdots<n^{\alpha} b_{n+1}<\cdots$.

Proof.

(i). We can get

$$
\frac{b_{j+1}}{b_{j}}=\frac{(j+1)^{\beta}-(j-1)^{\beta}}{(j+1)^{\beta}-j^{\beta}}=\frac{(1+2 / j)^{\beta}-(1+1 / j)^{\beta}}{(1+1 / j)^{\beta}-1} .
$$

Let $F(h):=\frac{(1+2 h)^{\beta}-(1+h)^{\beta}}{(1+h)^{\beta}-1}$. Then we obtain

$$
\begin{aligned}
F^{\prime}(h) & =\frac{\beta\left[\left(2(1+2 h)^{\beta-1}-(1+h)^{\beta-1}\right)\left((1+h)^{\beta}-\right)-\left((1+2 h)^{\beta}-(1+h)^{\beta}\right)(1+h)^{\beta-1}\right]}{\left((1+h)^{\beta}-1\right)^{2}} \\
& =\frac{\beta\left[2(1+2 h)^{\beta-1}(1+h)^{\beta}-2(1+2 h)^{\beta-1}+(1+h)^{\beta-1}-(1+2 h) \beta(1+h)^{\beta-1}\right]}{\left((1+h)^{\beta}-1\right)^{2}} \\
& =\frac{\beta\left[1+(1+2 h)^{1-\beta}-2(1+h)^{1-\beta}\right]}{\left((1+h)^{\beta}-1\right)^{2}(1+2 h)^{1-\beta}(1+h)^{1-\beta}} \\
& =\frac{\beta\left[\left((1+2 h)^{1-\beta}-(1+h)^{1-\beta}\right)-\left((1+h)^{1-\beta}-1\right)\right]}{\left((1+h)^{\beta}-1\right)^{2}(1+2 h)^{1-\beta}(1+h)^{1-\beta}} \\
& =\frac{\beta h(1-\beta)\left[1 /\left(1+h+\theta_{1}\right)^{\beta}-1 /\left(1+\theta_{2} h\right)^{\beta}\right]}{\left((1+h)^{\beta}-1\right)^{2}(1+2 h)^{1-\beta}(1+h)^{1-\beta}}<0,
\end{aligned}
$$

where $0<\theta_{1}, \theta_{2}<1$.

Therefore we can see that

$$
F(1 /(j-1))<F(1 / j)
$$

Consequently, we have

$$
\frac{b_{j}}{b_{j-1}} \leqslant \frac{b_{j+1}}{b_{j}} .
$$

(ii). In the case of $n=2 n^{\prime}, n^{\prime} \in N$, we get

$$
\bigcup_{j=1}^{n} b_{j} b_{n-j}=\left\{b_{1} b_{n-1}, b_{2} b_{n-2}, \ldots b_{n^{\prime}} b_{n^{\prime}}, b_{n^{\prime}+1} b_{n^{\prime}+1}, \ldots, b_{n} b_{0}\right\}=\left\{b_{0} b_{n}, b_{1} b_{n-1}, b_{2} b_{n-2}, \ldots, b_{n^{\prime}} b_{n^{\prime}}\right\} .
$$

On the other hand, using (2.13), for $k=\overline{1, n^{\prime}-1}$ we obtain

$$
\frac{b_{n^{\prime}-k}}{b_{n^{\prime}-k-1}} \leqslant \frac{b_{n^{\prime}-k+1}}{b_{n^{\prime}-k}}=\frac{b_{n^{\prime}-(k-1)}}{b_{n^{\prime}-k}} \leqslant \frac{b_{n^{\prime}-(k-2)}}{b_{n^{\prime}-(k-1)}} \leqslant \cdots \leqslant \frac{b_{n^{\prime}}}{b_{n^{\prime}-1}} \leqslant \frac{b_{n^{\prime}+1}}{b_{n^{\prime}}} \leqslant \cdots \leqslant \frac{b_{n^{\prime}+k+1}}{b_{n^{\prime}+k}} .
$$

Hence it is obvious that

$$
b_{n^{\prime}-k} \cdot b_{n^{\prime}+k} \leqslant b_{n^{\prime}-(k+1)} \cdot b_{n^{\prime}+(k+1)}
$$

holds.

Therefore, we get

$$
\min _{j}\left\{b_{j} b_{n-j}\right\}=b_{n^{\prime}}^{2}
$$


In the case of $n=2 n^{\prime}+1, n^{\prime} \in N$, we have

$\bigcup_{j=1}^{n} b_{j} b_{n-j}=\left\{b_{1} b_{n-1}, b_{2} b_{n-2}, \ldots, b_{n^{\prime}} b_{n^{\prime}+1}, b_{n^{\prime}+1} b_{n^{\prime}}, \ldots, b_{n} b_{0}\right\}=\left\{b_{0} b_{n}, b_{1} b_{n-1}, b_{2} b_{n-2}, \ldots, b_{n^{\prime}} b_{n^{\prime}+1}\right\}$

Using (2.13), we obtain

$$
\frac{b_{n^{\prime}}}{b_{n^{\prime}-1}} \leqslant \frac{b_{n^{\prime}+1}}{b_{n}^{\prime}} \leqslant \frac{b_{n^{\prime}+2}}{b_{n^{\prime}+1}}
$$

Thus, we can get

$$
b_{n^{\prime}} \cdot b_{n^{\prime}+1} \leqslant b_{n^{\prime}-1} \cdot b_{n^{\prime}+2} .
$$

Similarly to the case of $n=2 n^{\prime}$, it should be proved that

$$
\min _{j}\left\{b_{j} b_{n-j}\right\}=b_{n^{\prime}} \cdot b_{n^{\prime}+1} .
$$

(iii). Obviously, we have

$$
n^{\alpha} b_{n+1}=n^{\alpha}\left((n+2)^{1-\alpha}-(n+1)^{1-\alpha}\right)=\frac{(1+2 / n)^{1-\alpha}-(1+1 / n)^{1-\alpha}}{1 / n} .
$$

Let $E(h):=\frac{(1+2 h)^{1-\alpha}-(1+h)^{1-\alpha}}{h}$, then we get

$$
\begin{aligned}
E^{\prime}(h) & =\frac{\left[2(1-\alpha) /(1+2 h)^{\alpha}-(1-\alpha) /(1+h)^{\alpha}\right] h-\left[(1+2 h)^{1-\alpha}-(1+h)^{1-\alpha}\right]}{h^{2}} \\
& =\frac{\left[2(1-\alpha)-(1-\alpha)((1+2 h) /(1+h))^{\alpha}\right] h-\left[(1+2 h)-(1+h)((1+2 h) /(1+h))^{\alpha}\right]}{h^{2}(1+2 h)^{\alpha}} \\
& =\frac{[2(1-\alpha) h-(1+2 h)]+[(1+h)-(1-\alpha) h]((1+2 h) /(1+h))^{\alpha}}{h^{2}(1+2 h)^{\alpha}} \\
& =\frac{(-1-2 \alpha h)+(1+\alpha h)(1+h /(1+h))^{\alpha}}{h^{2}(1+2 h)^{\alpha}} .
\end{aligned}
$$

Since $(1+h /(1+h))^{\alpha}<1+\alpha h /(1+h)$, we have

$$
E^{\prime}(h)<\frac{(-1-2 \alpha h)+(1+\alpha h)(1+\alpha h /(1+h))}{h^{2}(1+2 h)^{\alpha}}=\frac{\alpha h(-1+(1+\alpha h) /(1+h))}{h^{2}(1+2 h)^{\alpha}}<0
$$

Thus it is obvious that $n^{\alpha} b_{n+1}$ is an increasing sequence.

The proof of Lemma 2.5 is completed.

Theorem 2.6. Inequality (2.10) holds if the following condition is satisfied:

$$
\frac{e \Gamma(2-\alpha) L}{2\left(3^{1-\alpha}-2^{1-\alpha}\right)^{2}} \leqslant 1
$$

Proof. We will prove this theorem in three steps.

(i). Inequality (2.10) holds if the following condition is satisfied.

$$
\tau_{k}^{2 \alpha-1} \leqslant \frac{1}{e \Gamma(2-\alpha) L} \min \left\{\sum_{j=1}^{n} b_{j}\left((n-j+1)^{1-\alpha}-(h-j)^{1-\alpha}\right), 1\right\}, \quad n=\overline{1, N_{k}}, k=\overline{0, m}
$$


In fact, from (2.15) we have

$$
\begin{aligned}
\tau_{k}^{\alpha} & \leqslant \frac{\tau_{k}^{1-\alpha}}{2 \Gamma(2-\alpha) L} \sum_{j=1}^{n} b_{j}\left((n-j+1)^{1-\alpha}-(n-j)^{1-\alpha}\right) \\
& =\frac{1}{e \Gamma(1-\alpha) L} \sum_{j=1}^{n} b_{j}\left(\left((n-j+1) \tau_{k}\right)^{1-\alpha}-\left((n-j) \tau_{k}\right)^{1-\alpha}\right) \\
& \leqslant \frac{1}{\Gamma(2-\alpha) L} \sum_{j=1}^{n} b_{j} e^{-\left(n \tau_{k}\right)^{1-\alpha}}\left(\left((n-j+1) \tau_{k}\right)^{1-\alpha}-\left((n-j) \tau_{k}\right)^{1-\alpha}\right) .
\end{aligned}
$$

Since $x>y>0 \rightarrow e^{x}-e^{y}>x-y$, we get

$$
\tau_{k}^{\alpha} \leqslant \frac{1}{\Gamma(2-\alpha) L} \sum_{j=1}^{n} b_{j} e^{-\left(n \tau_{k}\right)^{1-\alpha}}\left(e^{\left((n-j+1) \tau_{k}\right)^{1-\alpha}}-e^{\left((n-j) \tau_{k}\right)^{1-\alpha}}\right) .
$$

On the other hand, from (2.15) we can get

$$
\tau_{k}^{\alpha} \leqslant \frac{\tau_{k}^{1-\alpha}}{e \Gamma(2-\alpha) L} \leqslant \frac{\tau_{k}^{1-\alpha} e^{-\tau_{k}^{1-\alpha}}}{\Gamma(2-\alpha) L}=\frac{\left(2 \tau_{k}^{1-\alpha}-\tau_{k}^{1-\alpha}\right) e^{-\tau_{k}^{1-\alpha}}}{\Gamma(2-\alpha) L} \leqslant \frac{\left(e^{2 \tau_{k}^{1-\alpha}}-e^{\tau_{k}^{1-\alpha}}\right) e^{-\tau_{k}^{1-\alpha}}}{\Gamma(2-\alpha) L}=\frac{e^{\tau_{k}^{1-\alpha}}-1}{\Gamma(2-\alpha) L} .
$$

(ii). Inequality (2.15) holds if the following condition is satisfied.

$$
\tau_{k}^{2 \alpha-1} \leqslant \frac{2 N_{k}^{1-2 \alpha} b_{2}^{2}}{e \Gamma(2-\alpha) L}
$$

It is obvious that

$$
\sum_{j=1}^{n} b_{j}\left((n-j+1)^{1-\alpha}-(n-j)^{1-\alpha}\right)=\sum_{j=1}^{n} b_{j} b_{n-j} \geqslant n \cdot \min _{j}\left\{b_{j} b_{n-j}\right\} .
$$

Using Lemmas 2.4 and 2.5, we can get

$$
\min _{j}\left\{b_{j} b_{n-j}\right\} \geqslant b_{n^{\prime}+1}^{2}, n^{\prime}=[n / 2] .
$$

Thus we have

$$
\sum_{j=1}^{n} b_{j}\left((n-j+1)^{1-\alpha}-(n-j)^{1-\alpha}\right) \geqslant n b_{n^{\prime}+1}^{2} b_{n^{\prime}+1}^{2} \geqslant 2 n^{\prime} b_{n^{\prime}+1}^{2}
$$

If $n=1$, then we can see that

$$
\sum_{j=1}^{n} b_{j}\left((n-j+1)^{1-\alpha}-(n-j)^{1-\alpha}\right)=b_{1}>b_{2}>2 b_{2}^{2}>2 N_{k}^{1-2 \alpha} b_{2}^{2} .
$$

And if $n \geqslant 2$, by using Lemma 2.5 and (2.17) we get

$$
\sum_{j=1}^{n} b_{j}\left((n-j+1)^{1-\alpha}-(n-j)^{1-\alpha}\right) \geqslant 2 n^{\prime} b_{n^{\prime}+1}^{2}>2 n^{\prime} \cdot\left(n^{\prime}\right)^{-2 \alpha} b_{2}^{2}=2\left(n^{\prime}\right)^{1-2 \alpha} b_{2}^{2} \geqslant 2 N_{k}^{1-2 \alpha} b_{2}^{2} .
$$

On the other hand, since $\tau_{k}=T_{k} / N_{k} \leqslant 1$, we can see easily that

$$
\tau_{k}^{2 \alpha-1} \leqslant 1 \text {. }
$$

Therefore, if the inequality (2.16) is satisfied, then the expression (2.15) holds. 
(iii). Inequality (2.16) holds if the condition (2.14) is satisfied.

It can be easily shown that

$$
\tau_{k}^{2 \alpha-1} \leqslant\left(1 / N_{k}\right)^{2 \alpha-1}=N_{k}^{1-2 \alpha} \leqslant \frac{2 N_{k}^{1-2 \alpha} b_{2}^{2}}{e \Gamma(2-\alpha) L}
$$

Thus we can see that if the condition (2.14) is satisfied, then inequality (2.10) holds. This completes the proof.

Then we conclude the following.

Theorem 2.7. Assuming that the functions $\mathrm{f}$ and $\mathrm{I}_{\mathrm{k}}$ satisfy Lipschitz conditions (2.8)-(2.9)and the condition (2.14) is satisfied, the EDS defined by (2.6)-(2.7) is stable.

\subsection{Convergence of the effective difference scheme}

Let $x\left(t_{n}^{k}\right)$ be the exact solution of the problem (2.1) at mesh point $t_{n}^{k}$, and $x_{n}^{k}$ the numerical solution of the problem (2.1) computed by using the EDS (2.6)-(2.7).

Denote $\eta_{n}^{k}:=x\left(t_{n}^{k}\right)-x_{n}^{k}$.

Subtracting (2.6) from (2.5) leads to

$$
\eta_{n+1}^{k}=b_{n} \eta_{0}^{k}+\sum_{j=0}^{n-1}\left(b_{j}-b_{j+1}\right) \eta_{n-j}^{k}+\mu_{k}\left[f\left(t_{n}^{k}, x\left(t_{n}^{k}\right)\right)-f\left(t_{n}^{k}, x_{n}^{k}\right)\right]+R_{n+1}^{k}, \quad n=\overline{1, N_{k}-1, k=\overline{0, m} . ~}
$$

From the Lipschitz continuity of the function $f$, we get

$$
\left|\eta_{\mathfrak{n}+1}^{k}\right| \leqslant b_{n}\left|\eta_{0}^{k}\right|+\sum_{j=1}^{n-1}\left(b_{j}-b_{j+1}\right)\left|\eta_{n-j}^{k}\right|+\mu_{k} L\left|\eta_{n}^{k}\right|+\left|R_{n+1}^{k}\right| .
$$

Following a similar argument to that presented above for the stability analysis of the EDS (2.6)-(2.7), we have

$$
\begin{aligned}
\left|\eta_{1}^{k}\right| & \leqslant b_{0}\left|\eta_{0}^{k}\right|+\mu_{k} L\left|\eta_{0}^{k}\right|+\left|R_{1}^{k}\right|, \\
\left\|\eta_{1}^{k}\right\|_{1, k} & \leqslant\left\|\eta_{0}^{k}\right\|_{0}+e^{-\tau_{k}^{\beta}}\left|R_{1}^{k}\right|, \\
\left|\eta_{2}^{k}\right| & \leqslant b_{1}\left|\eta_{0}^{k}\right|+\left(b_{0}-b_{1}\right)\left|\eta_{1}^{k}\right|+\mu_{k} L\left|\eta_{1}^{k}\right|+\left|R_{2}^{k}\right|, \\
\left\|\eta_{2}^{k}\right\|_{2, k} & \leqslant b_{1} e^{-\left(2 \tau_{k}\right)^{\beta}}\left\|\eta_{0}^{k}\right\|_{0, k}+\left(b_{0}-b_{1}+\mu_{k} L\right) e^{-\left(2 \tau_{k}\right)^{\beta}+\tau_{k}^{\beta}}\left\|\eta_{1}^{k}\right\|_{1, k}+e^{-\left(2 \tau_{k}\right)^{\beta}}\left|R_{2}^{k}\right| \\
& \leqslant\left[b_{1} e^{-\left(2 \tau_{k}\right)^{\beta}}+\left(b_{0}-b_{1}+\mu_{k} L\right) e^{\left.-\left(2 \tau_{k}\right)^{\beta}+\tau_{k}^{\beta}\right]}\right]\left|\eta_{0}^{k} \|_{0, k}+\left(b_{0}-b_{1}+\mu_{k} L\right) e^{-\left(2 \tau_{k}\right)^{\beta}}\right| R_{1}^{k}\left|+e^{-\left(2 \tau_{k}\right)^{\beta}}\right| R_{2}^{k} \mid .
\end{aligned}
$$

Since from (2.10) the coefficients of $\left\|\eta_{0}^{k}\right\|_{0, k}$ and $e^{-\left(2 \tau_{k}\right)^{\beta}}\left|R_{1}^{k}\right|$ are smaller than 1 , we get

$$
\left\|\eta_{2}^{k}\right\|_{2, k} \leqslant\left\|\eta_{0}^{k}\right\|_{0, k}+e^{-\left(2 \tau_{k}\right)^{\beta}}\left(\left|R_{1}^{k}\right|+\left|R_{2}^{k}\right|\right) .
$$

By induction, it can be easily shown that

$$
\left\|\eta_{n}^{k}\right\|_{n, k} \leqslant\left\|\eta_{0}^{k}\right\|_{0, k}+e^{-\left(n \tau_{k}\right)^{\beta}} \sum_{j=1}^{n}\left|R_{i}^{k}\right| .
$$

Hence we can get

$$
\left\|\eta_{n}^{k}\right\|_{n, k} \leqslant\left\|\eta_{0}^{k}\right\|_{0, k}+e^{-\left(n \tau_{k}\right)^{\beta}} n c_{1} \tau_{k}^{1+\alpha} \leqslant\left\|\eta_{0}^{k}\right\|_{0, k}+n c_{1} \tau_{k}^{1+\alpha} \leqslant\left\|\eta_{0}^{k}\right\|_{0, k}+c_{1} \tau_{k}^{\alpha}
$$


Therefore, it is obvious that

$$
\max _{n}\left|\eta_{n}^{k}\right| \leqslant e\left(\left|\eta_{0}^{k}\right|+c_{1} \tau_{k}^{\alpha}\right)
$$

holds. In the case of $k=0$, by using $\eta_{0}^{0}=0$ we have

$$
\max _{n}\left|\eta_{n}^{0}\right| \leqslant c_{1} e \tau_{0}^{\alpha} .
$$

Let $\tau_{\max }:=\max _{k} \tau_{k}$. Then we obtain

$$
\max _{n}\left|\eta_{n}^{0}\right| \leqslant c_{1} e \tau_{\max }^{\alpha} .
$$

When $k \geqslant 1$, in the similar way to the above we get

$$
\begin{aligned}
\left|\eta_{0}^{1}\right| & \leqslant\left(1+L_{1}\right) c_{1} e \tau_{\text {max }}^{\alpha} \\
\max _{n}\left|\eta_{n}^{1}\right| & \leqslant e\left[\left(1+L_{1}\right) c_{1} e \tau_{\max }^{\alpha}+c_{1} \tau_{\text {max }}^{\alpha}\right]<\left(2+L_{1}\right) c_{1} e^{2} \tau_{\text {max }}^{\alpha}, \\
\max _{n}\left|\eta_{n}^{k}\right| & \leqslant 2\left(\left|\eta_{0}^{k}\right|+c_{1} \tau_{k}^{\alpha}\right) \leqslant e^{k+1} c_{1} \Pi_{i=1}^{k}\left(2+L_{i}\right) \cdot \tau_{\text {max }}^{\alpha} .
\end{aligned}
$$

Denote $c_{2}:=e^{m+1} c_{1} \Pi_{i=1}^{m}\left(2+L_{i}\right)$. Then we have

$$
\max _{n, k}\left|\eta_{n}^{k}\right| \leqslant c_{2} \tau_{\max }^{\alpha}
$$

Thus we conclude the following.

Theorem 2.8. Assuming that the functions $\mathrm{f}$ and $\mathrm{I}_{\mathrm{k}}$ satisfy the Lipschitz conditions (2.8)-(2.9) and inequality (2.14) holds, the EDS defined by (2.6)-(2.7) is convergent and there exists a positive constant $\mathrm{c}_{2}$ such that

$$
\max _{n, k}\left|\eta_{n}^{k}\right| \leqslant c_{2} \tau_{\max }^{\alpha}
$$

\section{I-type impulsive fractional differential equation and decomposition-operational matrix method}

\subsection{I-type impulsive fractional differential equation}

Definition 3.1. The equation (3.1) is said to be an I-type impulsive fractional differential equation.

$$
\left\{\begin{array}{l}
{ }^{c} D_{0}^{\alpha} x(t)=f(t, x(t)), t \in(0,1], t \neq t_{k} \in(0,1), \\
\left.\Delta x(t)\right|_{t=t_{k}}=I_{k}\left(x\left(t_{k}^{-}\right)\right), k=1,2, \ldots, l \\
x(0)=x_{0}
\end{array}\right.
$$

where

$$
\begin{aligned}
f & \in C(I \times X, X), \\
I_{k}: X & \rightarrow X, \\
0 & =t_{0}<t_{1}<\cdots<t_{l}<t_{l+1}=1, \\
\left.\Delta x(t)\right|_{t=t_{k}} & =x\left(t_{k}^{+}\right)-x\left(t_{k}^{-}\right), \\
x\left(t_{k}^{+}\right) & =\lim _{h \rightarrow+0} x\left(t_{k}+h\right), x\left(t_{k}^{-}\right)=\lim _{h \rightarrow+0} x\left(t_{k}-h\right) .
\end{aligned}
$$

Lemma 3.2. Assume that $\mathrm{g} \in \mathrm{C}(0,1) \cap \mathrm{L}(0,1)$ with a $\mathrm{n}^{\text {th }}$ derivative that belongs $\mathrm{C}(0,1) \cap \mathrm{L}(0,1)$. Then,

$$
\mathrm{I}_{0+}^{\alpha}{ }^{\mathrm{c}} \mathrm{D}_{\alpha+}^{\alpha}=\mathrm{g}(\mathrm{t})+\mathrm{c}_{1}+\mathrm{c}_{2} \mathrm{t}+\mathrm{c}_{3} \mathrm{t}^{2}+\cdots+\mathrm{c}_{\mathrm{n}} \mathrm{t}^{\mathrm{n}-1},
$$

where $c_{i} \in R, i=1, \ldots, n, n=[\alpha]+1$. 
Lemma 3.3. Let $\mathrm{q} \in(0,1)$ and $\mathrm{h}: \mathrm{J} \rightarrow \mathrm{R}$ be continuous. A function $\mu \in \mathrm{C}(\mathrm{J}, \mathrm{R})$ is a solution of the fractional integral equation

$$
\mu(t)=\mu_{0}-\frac{1}{\Gamma(q)} \int_{0}^{a}(a-s)^{q-1} h(s) d s+\frac{1}{\Gamma(q)} \int_{0}^{t}(t-s)^{q-1} h(s) d s,
$$

if and only if $\mu$ is a solution of the following fractional Cauchy problems

$$
\left\{\begin{array}{l}
{ }^{c} D_{t}^{q} \mu(t)=h(t), t \in J \\
\mu(a)=\mu_{0}, a>0
\end{array}\right.
$$

For the convenience, put $l=1$.

And then the problem we considered is

$$
\begin{aligned}
{ }^{c} D_{0}^{\alpha} \chi(t) & =f(t, x(t)), t \in(0,1], t \neq t_{8} \in(0,1), \\
\left.\Delta x(t)\right|_{t_{*}} & =I_{*}\left(x\left(t_{*}^{-}\right)\right), \\
x(0) & =x_{0}
\end{aligned}
$$

where $f \in C((0,1] \times R, R)$.

Operating the fractional integral operator $\mathrm{I}_{0}^{\alpha}$ on both sides of (3.2), we get

$$
I_{0}^{\alpha} \circ^{c} D_{0}^{\alpha} x(t)=I_{0}^{\alpha} \circ f(t, x(t)), t \in(0,1], t \neq t_{*} \in(0,1) .
$$

Then from $0<\alpha<1$ and Lemma 3.2 we have

$$
\mathrm{I}_{0+}^{\alpha}{ }^{\mathrm{c}} \mathrm{D}_{0}^{\alpha} x(\mathrm{t})=x(\mathrm{t})+\mathrm{c}_{1} .
$$

Therefore we can obtain

$$
\left.x(t)=I_{0}^{\alpha} \circ f(t, x(t))-c_{1}, t \in 90,1\right], t \neq t_{*} \in(0,1),
$$

where $x(t)$ satisfies (3.3) and (3.4).

When $t \in\left(0, t_{*}\right)$, from initial condition (3.4) we have

$$
x(0)=\left.I_{0}^{\alpha} \circ f(t, x(t))\right|_{t=0+}-c_{1}=x_{0} .
$$

Since $\left.I_{0}^{\alpha} \circ f(t, x(t))\right|_{t=0+}=0$, we get

$$
c_{1}=-x_{0}
$$

Thus, we have

$$
x(t)=I_{0}^{\alpha} f(t, x(t))+x_{0} .
$$

On the other hand, when $t \in\left(t_{*}, 1\right)$, from (3.3) we obtain

$$
\Delta x\left(t_{*}\right)=x\left(t_{*}^{+}\right)-x\left(t_{*}^{-}\right)=I_{*}\left(x\left(t_{*}^{-}\right)\right), \quad x\left(t_{*}^{+}\right)=x\left(t_{*}^{-}\right)+I_{*}\left(x\left(t_{*}^{-}\right)\right) .
$$

Using Lemma 3.3 we get

$$
x(t)=x_{0}+I_{*}\left(x\left(t_{*}^{-}\right)\right)+I_{0}^{\alpha} f(t, x(t)) .
$$

From (3.5) and (3.6) we can obtain the following integral equation

$$
x(t)=x_{0}+I_{*}\left(x\left(t_{*}^{-}\right)\right) x\left(t-t_{*}\right)+I_{0}^{\alpha} \circ f(t, x(t)),
$$

where $x(t)=\left\{\begin{array}{l}1, t>0 \\ 0, t \leqslant 0\end{array}, x_{0^{*}}:=\left\{\begin{array}{l}x_{0}, t<t_{*} \\ x_{*}^{-}, t>t_{*} .\end{array}\right.\right.$

Therefore we can see that if we can solve the integral equation (3.7), we can also get a solution of the problem (3.2)-(3.4).

The purpose of this paper is to propose a decomposition-operational matrix method that is a fast computing method by which we can compute a numerical solution of (3.7) at some discrete points. 


\subsection{Decomposition-operational matrix method}

$f, I_{*}$ are nonlinear functions, so we will use decomposition method.

$$
\text { (H3.1) } \quad x(t):=\sum_{n=0}^{\infty} x_{n}(t) .
$$

Equation (3.8) is satisfied if $f$ is an analytic function in respect to second parameter.

From (3.7) we get

$$
\sum_{n=0}^{\infty} x_{n}(t)=x_{0}+I_{*}\left(\sum_{n=0}^{\infty} x_{n}\left(t_{*}^{-}\right)\right) x\left(t-t_{*}\right)+I_{0}^{\alpha} \circ f\left(t, \sum_{n=0}^{\infty} x_{n}(t)\right) .
$$

And then we can rewrite above expression as

$$
\begin{aligned}
\sum_{n=0}^{\infty} x_{n}(t)= & x_{0}+I_{*}\left(\sum_{n=0}^{\infty} x_{n}\left(t_{*}^{-}\right)\right) \chi\left(t-t_{*}\right)+I_{0}^{\alpha} \circ f\left(t, \sum_{n=0}^{\infty} x_{n}(t)\right) x_{0} \\
& +\sum_{n=1}^{\infty}\left(I_{*}\left(\sum_{j=0}^{n} x_{j}\left(t_{*}^{-}\right)\right)-I_{*}\left(\sum_{j=0}^{n-1} x_{j}\left(t_{*}^{-}\right)\right)\right) \chi\left(t-t_{*}\right) \\
& +I_{*}\left(x_{0}\left(t_{*}^{-}\right)\right) \chi\left(t-t_{*}\right)+I_{0}^{\alpha} \circ \sum_{n=1}^{\infty}\left(f\left(t, \sum_{i=0}^{n} x_{j}(t)\right)-f\left(t, \sum_{i=0}^{n-1} x_{j}(t)\right)\right)+I_{0}^{\alpha} \circ f\left(t, x_{0}(t)\right) .
\end{aligned}
$$

From this we construct the following decomposition algorithm.

$$
\begin{aligned}
x_{0}(t) & =x_{0}, t \in(0,1] \\
x_{1}(t) & =I_{0}^{\alpha} \circ f\left(t, x_{0}(t)\right)+I_{*}\left(x_{0}\left(t_{*}^{-}\right)\right) \chi\left(t-t_{*}\right), \\
x_{n+1}(t) & =I_{0}^{\alpha} \circ\left(f\left(t, \sum_{j=0}^{n} x_{j}(t)\right)-f\left(t, \sum_{j=0}^{n-1} x_{j}(t)\right)\right)+\left(I_{*}\left(\sum_{j=0}^{n} x_{J}\left(t_{*}^{-}\right)\right)-I_{*}\left(\sum_{j=0}^{n-1} x_{j}\left(t_{*}^{-}\right)\right)\right) \chi\left(t-t_{*}\right), n=1,2, \ldots
\end{aligned}
$$

Define $\Delta t:=1 / m=1 / 2^{r}, r \in N, t_{k}:=(k-0.5) \Delta t, k=\overline{1, m}$. For the convenience, put $t_{*}=t_{m / 2}$.

Definition 3.4 ([8]). The m-set of Block-Pulse functions(BPF) is given by

$$
b_{i}(t)= \begin{cases}1, & \frac{i}{m} \leqslant t<\frac{i+1}{m}, i=\overline{0 . m-1} \\ 0, & \text { otherwise. }\end{cases}
$$

Definition $3.5([8])$. A pair of integers $(j, k)$ satisfying the following conditions is said to be a integer decomposition of index $i$.

$$
i=k+2^{j}-1, \quad 0 \leqslant j<i, \quad 1 \leqslant k<2^{j}+1 .
$$

Definition 3.6 ([9]). The Haar wavelet function is given by

$$
h_{0}(t)=\frac{1}{\sqrt{m}}, \quad h_{i}(t)=\frac{1}{\sqrt{m}} \begin{cases}2^{\frac{j}{2}}, & \frac{k-1}{2^{j}}<t \leqslant \frac{k-1 / 2}{2^{j}}, \\ -2^{\frac{j}{2}}, & \frac{k-1 / 2}{2^{j}}<t<\leqslant \frac{k}{2^{j}}, \\ 0, & \text { otherwise, }\end{cases}
$$

where $(j, k)$ is a integer decomposition of $i$. 
Definition 3.7. The Haar wavelet matrix for a set of collocation points $\left(t_{k}\right)$ is given by

$$
\mathrm{H}_{\text {matrix }}:=\left(\begin{array}{cccc}
h_{0}\left(t_{1}\right) & h_{0}\left(t_{2}\right) & \ldots & h_{0}\left(t_{m}\right) \\
h_{1}\left(t_{1}\right) & h_{1}\left(t_{2}\right) & \cdots & h_{1}\left(t_{m}\right) \\
\cdot & \cdot & \cdot & \cdot \\
\cdot & \cdot & \cdot & \cdot \\
\cdot & \cdot & \cdot & \cdot \\
h_{m-1}\left(t_{1}\right) & h_{m-1}\left(t_{2}\right) & \cdots & h_{m-1}\left(t_{m}\right)
\end{array}\right)
$$

Similarly we can define Block-Pulse function matrix. From the definition of Block-Pulse function, we can easily see that $B_{\text {matrix }}=I$.

Lemma $3.8([8])$. $\mathrm{F}_{\mathrm{B}}^{\alpha}$, the operational matrix of Block-Pulse function vector $\mathrm{B}(\mathrm{t})=\left(\mathrm{B}_{1}(\mathrm{t}), \mathrm{B}_{2}(\mathrm{t}), \ldots, \mathrm{B}_{\mathrm{m}}(\mathrm{t})\right)^{\mathrm{T}}$, is given by

$$
\mathrm{F}_{\mathrm{B}}^{\alpha}=\frac{1}{\mathrm{~m}^{\alpha}} \frac{1}{\Gamma(\alpha+2)}\left(\begin{array}{ccccc}
1 & \xi_{1} & \xi_{2} & \cdots & \xi_{\mathrm{m}-1} \\
0 & 1 & \xi_{1} & \cdots & \xi_{\mathrm{m}-2} \\
0 & 0 & 1 & \cdots & \xi_{\mathrm{m}-3} \\
\vdots & \vdots & \vdots & \vdots & \\
0 & 0 & \cdots & 0 & 1
\end{array}\right)
$$

where $\xi_{k}=(k+1)^{\alpha+1}-2 k^{\alpha+1}+(k-1)^{\alpha+1}$.

Theorem 3.9. $F_{h}^{\alpha}$, the operational matrix of Haar wavelet function vector $H(t)=\left(h_{0}(t), h_{1}(t), \ldots, h_{m-1}(y)\right)^{\top}$, is given by

$$
\mathrm{F}_{\mathrm{H}}^{\alpha}=\mathrm{H}_{\text {matrix }} \mathrm{F}_{\mathrm{B}}^{\alpha} \mathrm{H}_{\text {matrix }}^{\top}
$$

Proof. We have

$$
\exists C \in R^{m \times m} ; H(t)=C B(t) .
$$

Then we can easily get

$$
\mathrm{H}_{\text {matrix }}=\mathrm{CB}_{\text {matrix }}
$$

Since $B_{\text {matrix }}=I$, we have

$$
\mathrm{C}=\mathrm{H}_{\text {matrix }}
$$

We obtain

$$
I_{0}^{\alpha} H(t)=I_{0}^{\alpha} B(t)=C I_{0}^{\alpha} B(t)=C F_{B}^{\alpha} B(t)=H_{\text {matrix }} F_{B}^{\alpha} B(t), \quad I_{0}^{\alpha} H(t)=F_{H}^{\alpha} H(t) .
$$

And we get

$$
F_{H}^{\alpha} H(t)=H_{\text {matrix }} F_{B}^{\alpha} B(t) .
$$

Therefore we can see

$$
\mathrm{F}_{\mathrm{H}}^{\alpha} \mathrm{H}_{\text {matix }}=\mathrm{H}_{\text {matrix }} \mathrm{F}_{\mathrm{B}}^{\alpha} \mathrm{B}_{\text {matrix }}=\mathrm{H}_{\text {matrix }} \mathrm{F}_{\mathrm{B}}^{\alpha}, \quad \mathrm{F}_{\mathrm{H}}^{\alpha}=\mathrm{H}_{\text {matrix }} \mathrm{F}_{\mathrm{B}}^{\alpha} \mathrm{H}_{\text {matrix }}^{\top} .
$$

The proof is completed. 
Denote as follows:

$$
H_{m}(t):=\left(h_{0}(t), h_{1}(t), \ldots, h_{m-1}(t)\right)^{\top}, \quad C_{m}^{\top}:=\left(c_{0}, c_{1}, \ldots, c_{m-1}\right) .
$$

If $y(t)$ is a piecewise constant function, $y(t)$ can be approximated by a number of Haar functions. That is, we have

$$
y(t)=\sum_{k=0}^{m-1} c_{k} h_{k}(t)=C_{m}^{\top} H_{m}(t) .
$$

Therefore collocation equation is as follows:

$$
y\left(t_{k}\right)=C_{m}^{\top} H_{m}\left(t_{k}\right), k=\overline{1, m} .
$$

Put $Y:=\left(y\left(t_{1}\right), y\left(t_{2}\right), \ldots, y\left(t_{m}\right)\right)$. Then, we obtain

$$
Y=\left(y\left(t_{1}\right), y\left(t_{2}\right), \ldots, y\left(t_{m}\right)\right)=C_{m}^{\top}\left(H_{m}\left(t_{1}\right), h_{m}\left(t_{2}\right), \ldots, H_{m}\left(t_{m}\right)\right) .
$$

And we can rewrite this as

$$
\mathrm{Y}=\mathrm{C}_{\mathrm{m}}^{\mathrm{T}} \mathrm{H}_{\text {matrix}}
$$

So, we have

$$
\mathrm{C}_{\mathrm{m}}^{\mathrm{T}}=\mathrm{YH}_{\text {matrix}}^{\mathrm{T}}
$$

On the other hand, using $I_{0}^{\alpha} y(t)=I_{0}^{\alpha} C_{m}^{\top} H_{m}(t)=C_{m}^{\top} F_{H}^{\alpha} H_{m}(t)$, we can get

$$
\left(I_{0}^{\alpha} y\left(t_{1}\right), I_{0}^{\alpha} y\left(t_{2}\right), \ldots, I_{9}^{\alpha} y\left(t_{m}\right)\right)=C_{m}^{\top} F_{H}^{\alpha} H_{\text {matrix }} .
$$
by

Denote $Y:=\left(x_{0}, x_{0}, \ldots, x_{0}\right), C_{0}^{\top}:=Y \circ H_{\text {matrix }}^{\top}$. Then $\hat{x}_{0}(t)$, a collocation approximation of $x_{0}(t)$ is given

$$
\widehat{x}_{0}(t)=C_{0}^{\top} \circ H(t)
$$

Put as follows:

$$
\begin{aligned}
Y_{1} & :=\left(f\left(t_{1}, \hat{x}_{0}\left(t_{1}\right)\right), f\left(t_{2}, \hat{x}_{0}\left(t_{2}\right)\right), \ldots, f\left(t_{m}, \hat{x}_{0}\left(t_{m}\right)\right)\right), \\
Y_{2} & :=\left(I_{*}\left(\hat{x}_{0}\left(t_{1}\right)\right) \cdot \chi\left(t_{1}-t_{*}\right), I_{*}\left(\hat{x}_{0}\left(t_{2}\right)\right) \cdot \chi\left(t_{2}-t_{*}\right), \ldots, I_{*}\left(\hat{x}_{0}\left(t_{m}\right)\right) \cdot \chi\left(t_{m}-t_{*}\right),\right. \\
C_{1}^{\top} & :=Y_{1} H_{\text {matrix }}^{\top} F_{H}^{\alpha}+Y_{2} H_{\text {matrix }}^{\top} \\
\hat{x}_{1}(t) & :=C_{1}^{\top} \circ H(t) .
\end{aligned}
$$

Then $\hat{x}_{1}(t)$ is a collocation approximation of $x_{1}(t)$.

Put $\hat{x}_{n+1}(t):=C_{n+1}^{\top} \circ H(t)$. And then we have

$$
\begin{aligned}
\mathrm{U}_{\mathrm{n}+1}(t) & :=\sum_{j=0}^{n} \hat{x}_{j}(t)=\left(\sum_{j=0}^{n} C_{j}^{\top}\right) \cdot H(t), \\
\hat{x}_{n+1}(t) & =U_{n+1}(t)-U_{n}(t) \\
U_{n+1}(t) & =U_{n}(t)+I_{0}^{\alpha} \circ\left(f\left(t, U_{n}(t)\right)-f\left(t, U_{n-1}(t)\right)\right)+\left(I_{*}\left(U_{n}\left(t_{*}^{-}\right)\right)-I_{*}\left(U_{n-1}\left(t_{*}^{-}\right)\right)\right) x\left(t-t_{*}\right), n=1,2, \ldots
\end{aligned}
$$

Denote $S_{n+1}:=\sum_{j=0}^{n} C_{j}^{\top}$. From this we have

$$
\mathrm{U}_{\mathrm{n}+1}(\mathrm{t})=\mathrm{S}_{\mathrm{n}+1} \circ \mathrm{H}(\mathrm{t}) .
$$


Define $\overline{\mathrm{u}}_{\mathrm{n}+1}:=\left(\mathrm{U}_{\mathrm{n}+1}\left(\mathrm{t}_{1}\right), \ldots, \mathrm{U}_{\mathrm{n}+1}\left(\mathrm{t}_{\mathrm{m}}\right)\right)$. We can easily see that

$$
\overline{\mathrm{u}}_{\mathrm{n}+1}=\mathrm{S}_{\mathrm{n}+1} \circ \mathrm{H}_{\text {matrix }} \text {. }
$$

In order to obtain $P$ which satisfies that $I_{0}^{\alpha} \circ f\left(t, U_{n}(t)\right)=P \circ H(t)$, denote $f\left(t, U_{n}(t)\right)=\widetilde{C}^{\top} \circ H(t)$. Then we can get

$$
\left(f\left(t_{1}, u_{n}\left(t_{1}\right)\right), f\left(t_{2}, U_{n}\left(t_{2}\right)\right), \ldots, f\left(t_{m}, U_{n}\left(t_{m}\right)\right)\right)=\widetilde{C}^{\top} \circ H_{\text {matrix }}
$$

So we have

$$
\begin{aligned}
\widetilde{C}^{\top} & =\left(f\left(t_{1}, U_{n}\left(t_{1}\right)\right), f\left(t_{2}, U_{n}\left(t_{2}\right)\right), \ldots, f\left(t_{m}, U_{n}\left(t_{m}\right)\right)\right) \circ H_{\text {matrix }}^{\top}, \\
I_{0}^{\alpha} \circ f\left(t, U_{n}(t)\right) & =\widetilde{C}^{\top} \circ I_{0}^{\alpha} H(t)=\widetilde{C}^{\top} F_{H}^{\alpha} H(t), \\
I_{0}^{\alpha} \circ f\left(t, U_{n}(t)\right) & =\widetilde{C}^{\top} \circ I_{0}^{\alpha} H(t)=\widetilde{C}^{\top} F_{H}^{\alpha} H(t), \\
P=\widetilde{C}^{\top} F_{H}^{\alpha} & =\left(f\left(t_{1}, U_{n}\left(t_{1}\right)\right), f\left(t_{2}, U_{n}\left(t_{2}\right)\right), \ldots, f\left(t_{m}, U_{n}\left(t_{m}\right)\right)\right) \circ H_{\text {matrix }}^{\top} \circ F_{H}^{\alpha}, \\
I_{0}^{\alpha} \circ f\left(t, U_{n}(t)\right) & =\left(f\left(t_{1}, U_{n}\left(t_{1}\right)\right), f\left(t_{2}, U_{n}\left(t_{2}\right)\right), \ldots, f\left(t_{m}, U_{n}\left(t_{m}\right)\right)\right) \circ H_{\text {matrix }}^{\top} \circ F_{H}^{\alpha} \circ H(t),
\end{aligned}
$$

that is,

$$
\begin{aligned}
& \left(\left.I_{0}^{\alpha} f\left(t, u_{n}(t)\right)\right|_{t_{1}},\left.I_{0}^{\alpha} f\left(t, u_{n}(t)\right)\right|_{t_{2}}, \ldots,\left.I_{0}^{\alpha} f\left(t, U_{n}(t)\right)\right|_{t_{m}}\right) \\
& \quad=\left(f\left(t_{1}, u_{n}\left(t_{1}\right)\right), f\left(t_{2}, u_{n}\left(t_{2}\right)\right), \ldots, f\left(t_{m}, U_{n}\left(t_{m}\right)\right)\right) \circ H_{\text {matrix }}^{\top} \circ F_{H}^{\alpha} \circ H_{\text {matrix }} .
\end{aligned}
$$

For the convenience, denote

$$
F\left(\bar{u}_{n}\right):=\left(f\left(t_{1}, U_{n}\left(t_{1}\right)\right), f\left(t_{2}, U_{n}\left(t_{2}\right)\right), \ldots, f\left(t_{m}, U_{n}\left(t_{m}\right)\right)\right) \cdot I\left(\bar{u}_{n}\right):=I_{*}\left(U_{n}\left(t_{*}\right)\right) .
$$

On the other hand we should get $\mathrm{Q}^{\top}$ that satisfies

$$
\chi\left(t-t_{*}\right)=Q^{\top} \circ H(t) .
$$

Since $\left(\chi\left(t_{1}-t_{*}\right), \chi\left(t_{2}-t_{*}\right), \ldots, \chi\left(t_{m}-t_{*}\right)\right)=Q^{\top} \circ H_{\text {matrix }}$, we have

$$
\mathrm{Q}^{\top}=\left(\chi\left(\mathrm{t}_{1}-\mathrm{t}_{*}\right), \chi\left(\mathrm{t}_{2}-\mathrm{t}_{*}\right), \ldots, \chi\left(\mathrm{t}_{\mathrm{m}}-\mathrm{t}_{*}\right)\right) \circ \mathrm{H}_{\text {matrix }}^{\top}
$$

Thus, we get

$$
\chi\left(t-t_{*}\right)=\left(\chi\left(t_{1}-t_{*}\right), \chi\left(t_{2}-t_{*}\right), \ldots, \chi\left(t_{m}-t_{*}\right)\right) \circ H_{\text {matrix }}^{\top} \circ H(t) .
$$

Now, put $\bar{\chi}:=\left(\chi\left(t_{1}-t_{*}\right), \chi\left(t_{2}-t_{*}\right), \ldots, \chi\left(t_{m}-t_{*}\right)\right)$. Then we get

$$
\overline{\mathrm{U}}_{\mathrm{n}+1}=\overline{\mathrm{U}}_{\mathrm{n}}+\left(\mathrm{F}\left(\overline{\mathrm{U}}_{\mathrm{n}}\right)-\mathrm{F}\left(\overline{\mathrm{U}}_{\mathrm{n}-1}\right)\right) \circ \mathrm{H}_{\text {matrix }}^{\top} \circ \mathrm{H}_{\text {matrix }}^{\top} \circ \mathrm{F}_{\mathrm{H}}^{\alpha} \circ \mathrm{H}_{\text {matrix }}+\left(\mathrm{I}\left(\overline{\mathrm{U}}_{\mathrm{n}}\right)-\mathrm{I}\left(\overline{\mathrm{U}}_{\mathrm{n}-1}\right)\right) \circ \overline{\mathrm{X}} \text {. }
$$

From this, we have

$$
\begin{aligned}
S_{n+1} & =S_{n}+\left(F\left(\bar{u}_{n}\right)-F\left(\bar{u}_{n-1}\right)\right) \circ H_{\text {matrix }}^{\top} \circ F_{\text {matrix }}^{\alpha}+\left(I\left(\bar{u}_{n}\right)-I\left(\bar{U}_{n-1}\right)\right) \circ \bar{\chi} \circ H_{\text {matrix }}^{\top} \\
F\left(\bar{U}_{n}\right) & =\left(f\left(t_{1}, U_{n}\left(t_{1}\right)\right), f\left(t_{2}, u_{n}\left(t_{2}\right)\right), \ldots, f\left(t_{m}, U_{n}\left(t_{m}\right)\right)\right) .
\end{aligned}
$$

Considering that $\mathrm{U}_{\mathrm{n}}\left(\mathrm{t}_{\mathrm{i}}\right)=\mathrm{S}_{\mathrm{n}} \circ \mathrm{H}\left(\mathrm{t}_{\mathrm{i}}\right)=\mathrm{S}_{\mathrm{n}} \circ \mathrm{H}_{\text {matrix }}^{\mathrm{i}}$, we can obtain

$$
F\left(\bar{U}_{n}\right)=\left(f\left(t_{1}, S_{n} \circ H_{\text {matrix }}^{1}\right), f\left(t_{2}, S_{n} \circ H_{\text {matrix }}^{2}\right), \ldots, f\left(t_{m}, S_{n} \circ H_{\text {matrix }}^{m}\right)\right)=: \bar{F}\left(S_{n}\right) .
$$

Similarly, we have

$$
\mathrm{I}\left(\overline{\mathrm{U}}_{\mathrm{n}}\right)=\mathrm{I}_{*}\left(\mathrm{U}_{\mathrm{n}}\left(\mathrm{t}_{*}\right)\right)=\mathrm{I}_{*}\left(\mathrm{~S}_{\mathrm{n}} \circ \mathrm{H}\left(\mathrm{t}_{*}\right)\right) .
$$


Using the assumption that $t_{*}=t_{m / 2}$, we get

$$
\mathrm{I}\left(\overline{\mathrm{U}}_{\mathrm{n}}\right)=\mathrm{I}_{*}\left(\mathrm{~S}_{\mathrm{n}} \circ \mathrm{H}_{\text {matrix }}^{\mathrm{m} / 2}\right)=: \overline{\mathrm{I}}\left(\mathrm{S}_{\mathrm{n}}\right) .
$$

Therefore, we have

$$
S_{n+1}=S_{n}+\left(\bar{F}\left(S_{n}\right)-\bar{F}\left(S_{n-1}\right)\right) \circ H_{\text {matrix }}^{\top} \circ F_{H}^{\alpha}+\left(\bar{I}\left(S_{n}\right)-\bar{I}\left(S_{n-1}\right)\right) \circ \bar{\chi} \circ H_{\text {matrix }}^{\top}
$$

Algorithm (decomposition-operational matrix method):

Step 1. Compute $S_{0}:=C_{0}^{\top}=\left(x_{0}, x_{0}, \ldots, x_{0}\right) \circ H_{\text {matrix}}^{\top}$.

Step 2. Compute $S_{1}:=C_{0}^{\top}+C_{1}^{\top}=C_{0}^{\top}+\left(Y_{1} H_{\text {matrix }}^{\top} F_{H}^{\alpha}+Y_{2} H_{\text {matrix }}^{\top}\right)$, where

$$
\begin{aligned}
\widehat{x}_{0}(t) & =C_{0}^{\top} \circ H_{\text {matrix }}, \\
Y_{1} & :=\left(f\left(t_{1}, \hat{x}_{0}\left(t_{1}\right)\right), f\left(t_{2}, \hat{x}_{0}\left(t_{2}\right)\right), \ldots, f\left(t_{m}, \hat{x}_{0}\left(t_{m}\right)\right)\right), \\
Y_{2} & :=\left(I_{*}\left(\hat{x}_{0}\left(t_{1}\right)\right) \cdot \chi\left(t_{1}-t_{*}\right), I_{*}\left(\hat{x}_{0}\left(t_{2}\right)\right) \cdot \chi\left(t_{2}-t_{*}\right), \ldots, I_{*}\left(\hat{x}_{0}\left(t_{m}\right)\right) \cdot \chi\left(t_{m}-t_{*}\right) .\right.
\end{aligned}
$$

Step 3. Compute $\mathrm{N}$ times

$$
S_{n+1}=s_{n}+\left(\bar{F}\left(S_{n}\right)-\bar{F}\left(S_{n-1}\right)\right) \circ H_{\text {matrix }}^{\top} F_{H}^{\alpha}+\left(\bar{I}\left(S_{n}\right)-\bar{I}\left(S_{n-1}\right) \circ \bar{\chi} \circ H_{\text {matrix }}^{\top} .\right.
$$

Step 4. Compute $\overline{\mathrm{U}}_{\mathrm{n}}=\mathrm{S}_{\mathrm{n}} \circ \mathrm{H}_{\text {matrix }}$.

3.3. Convergence of the approximate solution

Lemma 3.10 ([6]). Assume that $M:=\sup |f(t, x)|, t_{2}>t_{1}$. Then the following inequality holds:

$$
\left|I_{0}^{\alpha} f(t, x(t))\right|_{t_{1}}-\left.I_{0}^{\alpha} f(t, x(t))\right|_{t_{2}} \mid \leqslant \begin{cases}\frac{2 M}{\Gamma(1+\alpha)} \Delta t^{\alpha}, & \alpha \leqslant 1, \\ \frac{M}{\Gamma(1+\alpha)}\left(\left(t_{2}-t_{1}\right)^{\alpha}+t_{2}^{\alpha}-t_{1}^{\alpha}\right), & \alpha>1 .\end{cases}
$$

H 3.2 $\forall x_{1}, x_{2} \in R,\left|f\left(t, x_{1}\right)-f\left(t, x_{2}\right)\right| \leqslant L_{f}\left|x_{1}-x_{2}\right|$.

H $3.3 \forall x 1, x_{2} \in R,\left|I_{*}\left(x_{1}\right)-I_{*}\left(x_{2}\right)\right| \leqslant L_{I}\left|x_{1}-x_{2}\right|$.

H $3.4 \omega:=\frac{\mathrm{L}_{f}}{\Gamma(1+\alpha)}+\mathrm{L}_{\mathrm{I}_{*}}, 0<\omega<1$.

Theorem 3.11. The following inequality holds.

$$
\left\|x-U_{N}\right\|_{p c[0,1]} \leqslant \frac{2 M}{\Gamma(1+\alpha)(1-\omega)}\left(\frac{\Delta t}{2}\right)^{\alpha}+\omega^{N}\left\|x-U_{0}\right\|_{p c[0,1]} .
$$

Proof. Put $\forall t \in[0,1], \exists t_{k}: t_{k} \in U_{\Delta t}(t),\left|x(t)-U_{N}(t)\right|=\left|x(t)-x\left(t_{k}\right)+x\left(t_{k}\right)-U_{N}\left(t_{k}\right)+U_{N}\left(t_{k}\right)-U_{N}(t)\right|$. From the construction of $U_{N}(t)$, we get

$$
\left|\mathrm{u}_{\mathrm{N}}\left(\mathrm{t}_{\mathrm{k}}\right)-\mathrm{u}_{\mathrm{N}}(\mathrm{t})\right|=\left|\mathrm{u}_{\mathrm{N}}\left(\mathrm{t}_{\mathrm{k}}\right)-\mathrm{u}_{\mathrm{N}}\left(\mathrm{t}_{\mathrm{k}}\right)\right|=0 .
$$

We can easily see that

$$
x(t)=x_{0}+I_{*}\left(x\left(t_{*}^{-}\right)\right) \chi\left(t-t_{*}\right)+I_{0}^{\alpha} \circ f(t, x(t)), \quad x\left(t_{k}\right)=x_{0}+I_{*}\left(x\left(t_{*}^{-}\right)\right) \chi\left(t_{k}-t_{*}\right)+\left.I_{0}^{\alpha} \circ f(t, x(t))\right|_{t_{k}} .
$$

Using $t_{k} \in \bigcup_{\frac{\Delta}{2}}(t)$ and Lemma 3.10, we have

$$
\left|x(t)-x\left(t_{k}\right)\right|=\left|I_{0}^{\alpha} \circ f(t, x(t))-I_{0}^{\alpha} \circ f(t, x(t))\right|_{t_{k}} \mid \leqslant \frac{2 M}{\Gamma(1+\alpha)}\left(\frac{\Delta t}{2}\right)^{\alpha} .
$$


On the other hand, we have

$$
\begin{aligned}
\mathrm{U}_{\mathrm{N}}\left(\mathrm{t}_{\mathrm{k}}\right) & =\sum_{j=0}^{\mathrm{N}} \widehat{x}_{j}\left(t_{k}\right)=x_{0}+\mathrm{I}_{0}^{\alpha} f\left(t, \sum_{j=0}^{N-1} \widehat{x}_{j}\left(t_{k}\right)\right)+\mathrm{I}_{*}\left(\sum_{j=0}^{N-1} \hat{x}_{j}\left(t_{*}^{-}\right)\right) \chi\left(t_{k}-t_{*}\right) \\
& =x_{0}+I_{0}^{\alpha} f\left(t, u_{N-1}\left(t_{k}\right)\right)+I_{*}\left(U_{N-1}\left(t_{*}^{-}\right)\right) \chi\left(t_{k}-t_{*}\right) .
\end{aligned}
$$

Thus, we obtain the following estimate inequality:

$$
\begin{aligned}
\left|x\left(t_{k}\right)-U_{N}\left(t_{k}\right)\right| & =\left|I_{0}^{\alpha}\left(f(t, x(t))-f\left(t, U_{N-1}(t)\right)\right)\right|_{t_{k}}|+| I_{*}\left(x\left(t_{*}^{-}\right)\right)-I_{*}\left(U_{N-1}\left(t_{*}^{-}\right)\right) \mid \\
& \leqslant \frac{L_{f}}{\Gamma(1+\alpha)}\left\|x-U_{N-1}\right\|_{p c[0,1]}+L_{I_{*}}\left\|x-U_{N-1}\right\|_{p c[0,1]}, \\
\left|x(t)-U_{N}(t)\right| & \leqslant \frac{2 M}{\Gamma(1+\alpha)}\left(\frac{\Delta t}{2}\right)^{\alpha}+\frac{L_{f}}{\Gamma(1+\alpha)}\left\|x-U_{N-1}\right\|_{p c[0,1]}+L_{I_{*}}\left\|x-U_{N-1}\right\|_{p c[0,1]} \\
& =\frac{2 M}{\Gamma(1+\alpha)} \Delta t^{\alpha}+\omega\left\|x-U_{N-1}\right\|_{p c[0,1]} \leqslant \cdots \leqslant \frac{2 M}{\Gamma(1+\alpha)(1-\omega}\left(\frac{\Delta t}{2}\right)^{\alpha}+\omega^{N}\left\|x-U_{0}\right\|_{p c[0,1]} .
\end{aligned}
$$

Therefore we get

$$
\left\|x-U_{N}\right\|_{p c[0,1]} \leqslant \frac{2 M}{\Gamma(1+\alpha)(1-\omega)}\left(\frac{\Delta t}{2}\right)^{\alpha}+\omega^{N}\left\|x-U_{0}\right\|_{p c[0,1]} .
$$

The proof is completed.

\subsection{Numerical example}

\subsubsection{Problem}

Consider the following nonlinear impulsive fractional differential equation:

$$
{ }^{c} D_{0}^{\alpha} x(t)=f(t, x(t)), t \in(0,1], t \neq t_{*}=1 / 2, \quad \Delta x\left(t_{*}\right)=-0.2 x\left(t_{*}^{-}\right), \quad x(0)=1,
$$

where $\alpha=0.8$.

Put $f(t, x(t))$ as follows:

$$
\begin{aligned}
g_{1}(t) & =\left(2(2 t-1)^{-\alpha}\left(4(2-1 / t)^{\alpha} t^{2}+2^{\alpha}(-1+\alpha-2 t)(-1+2 t)\right)\right) /(4 \Gamma(1-\alpha)(2+(-3+\alpha) \alpha)), \\
g_{2}(t) & =\left(-2^{-2+\alpha} 3(-1+2 t)^{3-\alpha}\right) /\left(\Gamma(1-\alpha)^{*}\left(6-11 \alpha+6 \alpha^{2}-\alpha^{3}\right)\right), \\
f_{1}(t) & = \begin{cases}2 t^{(2-\alpha)} / \Gamma(3-\alpha), & t \leqslant \frac{1}{2} \\
g_{1}(t)+g_{2}(t), & t>\frac{1}{2}\end{cases} \\
f_{2}(t) & = \begin{cases}\left(t^{2}+1\right)^{2}, & t \leqslant \frac{1}{2} \\
\left(1-(t-1 / 2)^{3}\right)^{2}, & t>\frac{1}{2}\end{cases} \\
f(t, x(t)) & =x^{2}(t)+f_{1}(t)-f_{2}(t) .
\end{aligned}
$$

\subsubsection{Results of computation}

We put $r=7$ and divided [0,1] into $2^{r}=2^{7}=128$ equal parts.

The mesh points were taken as the midpoints of all subintervals and we used the $11^{\text {th }}$ order of decomposition.

The exact solution of this equation is as follows:

$$
x(t)= \begin{cases}t^{2}+1, & t \leqslant \frac{1}{2} \\ 1-(t-1 / 2)^{3}, & t>\frac{1}{2}\end{cases}
$$


Table 1: The maximum norm error of the exact solution and approximate solution.

\begin{tabular}{|c|c|c|c|c|c|c|c|c|c|c|c|c|c|c|c|c|}
\hline & & nate & Exact & Enror & Mesh Point & Approximate & Exact & Error & Mesh Point & Approximate & Exact & Error & Mesh Point & Approximate & Exact & Error \\
\hline 1 & 0.00391 & 1.00003 & 1.00002 & $1.38471 \mathrm{E}-05$ & 0.253906 & 1.03449 & 1.06447 & $2.61114 \mathrm{E}-\mathrm{c} 5$ & 0.503906 & 1.0003 & 1 & 0.0003026 & 0.753906 & $0.98<927$ & 0.98363 & 0. 3012963 \\
\hline 2 & 0.01172 & 1.00015 & 1.00014 & 0.000013649 & 0.261719 & 1.03852 & 1.0685 & 2. $66832 \mathrm{E}-\mathrm{C5}$ & 0.511719 & 1. 00049 & 999998 & 0.0004957 & 0.761719 & 0.983396 & 0.98207 & 0.001323 \\
\hline 3 & 0.01953 & 1.0004 & 1.00038 & 1. $38303 \mathrm{E}-05$ & 0.269531 & 1.07267 & $1.0^{\top} 265$ & 2. $72693 \mathrm{E}-\mathrm{c} 5$ & 0.519531 & 1.00055 & 0.999993 & 0.0005556 & 0.769531 & 0.981769 & 0.98042 & 0. 3013499 \\
\hline 4 & 0.02731 & 1. 00076 & 1.00075 & $1.40846 \mathrm{E}-05$ & $0.2773<4$ & 1.07695 & $1.0^{\top} 692$ & 2. $78703 \mathrm{E}-\mathrm{c} 5$ & 0.527344 & 1.00058 & 0.99998 & 0.000596 & 0.777344 & 0.980044 & 0.97867 & 0. 3013771 \\
\hline 5 & 0.03513 & 1.00125 & I. 00124 & $1.43693 \mathrm{E}-05$ & 0.285156 & 1.03134 & 1.08131 & 2. $84867 \mathrm{~T}-\mathrm{C} 5$ & 0.535156 & 1. 00059 & 0.999957 & 0.0006285 & 0.785156 & 0.978218 & 0.97681 & 0. J014047 \\
\hline 6 & 0.04297 & 1. 00186 & 1.00185 & $1.46^{\top} 13 \mathrm{E}-05$ & 0.292969 & 1.03586 & 1.08583 & 0.000029119 & 0.542969 & 1.00058 & 0.999921 & 0.000657 & 0.792969 & 0.976287 & 0.97485 & 0. 3014327 \\
\hline 7 & 0.05073 & 1. 00259 & 1.00258 & $1.49853 \mathrm{E}-05$ & 0.300781 & 1. 3905 & 1.09047 & $0.0000297 € 8$ & 0.550781 & 1.00055 & 0.999869 & 0.0006832 & 0.800781 & $0.97 \measuredangle 249$ & 0.97273 & 0. 3014609 \\
\hline 8 & 0.05853 & 1.00345 & 1.00343 & 1. $53089 \mathrm{E}-05$ & 0.308594 & 1.03526 & 1.09523 & $0.0000304: 4$ & 0.558594 & 1.00051 & 0.999799 & 0.0007078 & 0.808594 & 0.972102 & 0.97061 & 0.3014895 \\
\hline 9 & 0.06641 & 1.00443 & 1. 00441 & 1. $5640 \mathrm{TE}-05$ & & 1.13014 & 1.10011 & $3.11179 \mathrm{E}-\mathrm{C} 5$ & & & & 0.0007316 & & & & \\
\hline 10 & 0.07422 & 1. 00552 & 1. 00551 & 1. $59801 \mathrm{E}-05$ & 0.324219 & 1.13515 & 1.10512 & 3. $18202 \mathrm{E}-\mathrm{C} 5$ & 0.574219 & 1.00035 & 0.999591 & 0.0007546 & 0.824219 & 0.967466 & 0.96592 & 0. 3015476 \\
\hline 11 & 0.08203 & 1. 00675 & 1. 00673 & $1.63268 \mathrm{E}-05$ & 0.332031 & 1. 11028 & $1.1: 024$ & 3. $25415 \mathrm{E}-\mathrm{c} 5$ & 0.582031 & & $0.999<48$ & 0.0 & & $0.96 \leq 972$ & 0.9631 & \\
\hline 12 & 0.08981 & 1.00809 & 1.00807 & $1.66806 \mathrm{E}-05$ & 0.339844 & 1.11553 & $1.1: 549$ & 3. $32827 \mathrm{E}-\mathrm{C5}$ & 0.589844 & 1.00007 & 0.999275 & 0.0007996 & 0.839844 & 0.962357 & 0.96075 & 0.001607 \\
\hline 13 & 0.09763 & 1. 00955 & 1.00954 & 1. $70415 \mathrm{E}-05$ & 0.347656 & 1.1209 & 1.12086 & $3.40443 \mathrm{E}-\mathrm{c} 5$ & 0.597656 & 0.999891 & 0.999069 & 0.0008218 & 0.847656 & 0.959618 & 0.95793 & 0. 3016372 \\
\hline 14 & 0.10547 & 1.01114 & 1.01112 & 1. $7409 \mathrm{~F}-05$ & 0.355469 & 1.12639 & 1.12636 & $3.48272 \mathrm{E}-\mathrm{c} 5$ & 0.605469 & .999671 & 0.998827 & 0.000844 & 0.855469 & 0.956751 & 0.95503 & 0. 3016676 \\
\hline 15 & 0.11323 & 1.01285 & 1.01283 & $1.77853 \mathrm{E}-05$ & 0.363281 & 1.13201 & 1.13197 & 3. $56321 \mathrm{E}-\mathrm{c} 5$ & 0.613281 & 0.999412 & 0.998546 & 0.0008661 & 0.863281 & 0.953755 & 0.95203 & 0. 3016984 \\
\hline 16 & 0.12103 & 1. 01468 & 1.01466 & $1.8168 \leq \mathrm{E}-05$ & 0.371094 & 1.13775 & 1.13771 & 3. $64598 \mathrm{E}-\mathrm{c} 5$ & 0.621094 & 0.999113 & 0.998224 & 0.0008882 & 0.871094 & 0.950626 & 0.9483 & 0. 3017294 \\
\hline 17 & 0.12891 & 1.01664 & 1.01662 & $1.85593 \mathrm{E}-05$ & 0.378906 & 1.14361 & 1.14357 & 0.000037311 & 0.628906 & 0.998768 & 0.997858 & 0.0009105 & 0.878906 & $0.94 i 361$ & 0.9453 & 0. J017607 \\
\hline 18 & 0.13672 & 1. 01871 & 1.01869 & $1.89582 \mathrm{E}-05$ & 0.386719 & 1.14959 & 1.14955 & $3.81867 \mathrm{E}-\mathrm{C5}$ & 0.636719 & 0.998377 & $0.997 \measuredangle 44$ & 0.0009328 & 0.886719 & 0.943958 & 0.94217 & 0. 3017923 \\
\hline 19 & 0.14453 & 1.02091 & 1.02089 & $1.93653 \mathrm{E}-05$ & 0.394531 & 1. 15569 & 1.15565 & 3. $90876 \mathrm{E}-\mathrm{C} 5$ & 0.644531 & 0.997936 & 0.996981 & 0.0009553 & 0.894531 & 0.940413 & 0.93853 & 0. J018241 \\
\hline 20 & 0.15234 & 1.02323 & 1.02321 & 0.0000 & $0.4023<4$ & 1. 13192 & 1.16188 & 4. $00147 \mathrm{E}-\mathrm{C5}$ & 344 & & $0.996<64$ & 0.000978 & 0. & 0.936725 & 0.93487 & 0. \\
\hline 21 & 0.16013 & 1.02567 & 1.02565 & 2. $02055 \mathrm{E}-05$ & 0.410156 & 1.13827 & 1.16823 & 4. $09687 \mathrm{E}-\mathrm{C5}$ & 0.660156 & 0.996893 & 0.995892 & 0.0010009 & 0.910156 & 0.932889 & 0.931 & 0. 3018885 \\
\hline 22 & 0.16797 & 1.02823 & 1. 02821 & 2. $06392 \mathrm{E}-05$ & 0.417969 & 1.17474 & 1. : 747 & 4. $19506 \mathrm{E}-\mathrm{C} 5$ & 0.667969 & 0.996285 & 0.995261 & 0.001024 & 0.917969 & 0.928903 & 0.92693 & 0.001921 \\
\hline 23 & 0.17573 & 1. 03092 & 1.0309 & 2. $10822 \mathrm{E}-05$ & 0.425781 & 1.13133 & 1.18129 & 4. $29613 \mathrm{E}-\mathrm{c} 5$ & 0.675781 & & 0.994569 & 0.0010474 & & & 0.92281 & \\
\hline 24 & 0.18353 & 1.03373 & 1.03371 & 2. $15351 \mathrm{E}-05$ & 0.433594 & 1.13805 & 1.188 & 4. $40016 \mathrm{E}-\mathrm{C5}$ & 0.683594 & 4883 & 0.993812 & 0.001071 & 0.933594 & 10.9 & 0.91843 & 0. 3019866 \\
\hline 25 & 0.19141 & 1. 03666 & 1. 03664 & 0.000021998 & 0.441406 & & 1. 19484 & 4. $50723 \mathrm{E}-\mathrm{c} 5$ & & & & 0.0010949 & & & & \\
\hline 26 & 0.19922 & 1.03971 & 1. 03969 & 2. $24^{\top} 1 \leqslant \mathrm{E}-05$ & 0.449219 & 1. $2 J 184$ & 1.2018 & 4. $61745 \mathrm{E}-\mathrm{C5}$ & 0.699219 & 0.993212 & 0.992093 & 0.001119 & 0.949219 & 0.911402 & $0.9093 \overline{3}$ & 0. 3020529 \\
\hline 27 & 0.20703 & 1.04288 & 1.04286 & 2. $2955 \mathrm{iE}-05$ & 0.457031 & 1. $2 J 892$ & 1.20888 & 4. $73088 \mathrm{E}-15$ & 0.707031 & 0.99227 & 0.991126 & 0.0011434 & 0.957031 & 0.906623 & 0.90454 & 0. J020862 \\
\hline 28 & 0.21481 & 1.04618 & 104616 & 2. $34512 \mathrm{E}-05$ & $0.1648 \mathrm{a}$ & 121613 & $1.2: 608$ & $0.0000484 i 6$ & & & & 0.001 & 0.964844 & 0.901676 & 0.89953 & 0.3021196 \\
\hline 29 & 0.22263 & 1.0496 & 1. 04958 & 2. $39583 \mathrm{E}-05$ & 0.472656 & 1.22345 & 1.2234 & 4. $96769 \mathrm{E}-\mathrm{C} 5$ & 0.722656 & 0.990155 & 0.988962 & 0.0011931 & 0.972656 & 0.89656 & 0.89441 & 0. 3021532 \\
\hline 30 & 0.23047 & 1. 05314 & 1.05312 & $2.44^{\top} 7 \leqslant \mathrm{E}-05$ & 0.480469 & 1.2309 & 1.23085 & 0.000050912 & 0.730469 & 0.988977 & $0.987 i 58$ & 0.0012185 & 0.980469 & 0.89127 & 0.88903 & 0. 3021868 \\
\hline 31 & 0.23823 & 1.0568 & 1.05678 & 0.000025009 & 0.488281 & 1.23847 & 1.23842 & $5.21819 \mathrm{E}-\mathrm{C} 5$ & 0.738281 & 0.987715 & $0.986 \leq 71$ & 0.0012441 & 0.988281 & 0.885805 & 0.88353 & 0. 3022204 \\
\hline 32 & 0.24603 & 1. 06059 & 1. 06056 & 2. $55535 \mathrm{E}-05$ & 0.496094 & 1. 24616 & 1.24611 & 5. $34869 \mathrm{E}-\mathrm{C} 5$ & 0.746094 & 0.986366 & 0.985096 & 0.0012701 & 0.996094 & 0.880161 & 0.87791 & 0. 3022541 \\
\hline
\end{tabular}

Figure 1 shows the exact solution and approximate solution.

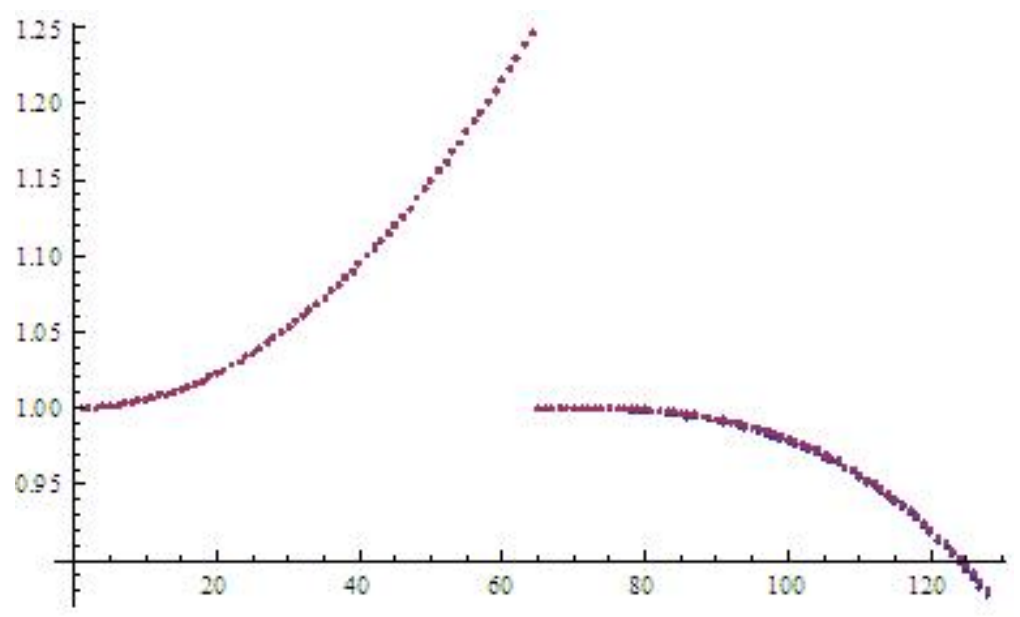

Figure 1: Exact solution and approximate solution

\section{Acknowledgment}

This work was supported by National Natural Science Foundation of China (No. 11761074 and No. 11361066), the projection of the Department of Science and Technology of JiLin Province (No. 20170101052JC) and the Educational Commission of Jilin Province of China (No. JJKH20170453KJ) and natural science foundation of Liaoning Province (No. 201602547).The authors are grateful to the referees for their useful suggestions which improved the quality and the scientific results of this paper.

\section{References}

[1] B. Ahmad, S. Sivasundaram, Existence results for nonlinear impulsive hybrid boundary value problems involving fractional differential equations, Nonlinear Anal. Hybrid Syst., 3 (2009), 251-258. 1 
[2] K. Balachandran, S. Kiruthika Existence of solutions of abstract fractional impulsive semilinear evolution equations, Electron. J. Qual. Theory Differ. Equ., 2010 (2010), 12 pages. 1

[3] M. Belmekki, J. J. Nieto, R. Rodrguez-Lpez, Existence of solution to a periodic boundary value problem for a nonlinear impulsive fractional differential equation, Electron. J. Qual. Theory Differ. Equ., 2014 (2014), 27 pages. 1

[4] M. Benchohra, D. Seba, Impulsive fractional differential equations in Banach spaces, Electron. J. Qual. Theory Differ. Equ., 2009 (2009), 14 pages. 1

[5] Y. Chang, A. Anguraj, P. Karthikeyan, Existence results for initial value problems with integral condition for impulsive fractional differential equations, J. Fract. Calc. Appl., 7 (2012), 1-10. 1

[6] K. Diethelm, The analysis of fractional differential equations, Springer-Verlag, Berlin, (2010). 1, 3.10

[7] M. Feckan, Y. Zhou, J. R. Wang, On the concept and existence of solution for impulsive fractional differential equations, Commun. Nonlinear Sci. Numer. Simul., 17 (2012), 3050-3060. 1

[8] A. K. Gupta, S. S. Ray, Wavelet Methods for Solving Fractional Order Differential Equations, Math. Probl. Eng., 2014 (2014), 11 pages. 3.4, 3.5, 3.8

[9] G. Hariharan, K. Kannan, An Overview of Haar Wavelet Method for Solving Differential and Integral Equations, World Appl. Sci. J., 23 (2013), 1-14. 1, 3.6

[10] V. Lakshmikantham, S. Leela, D. J. Vasundhara, Theory of fractional dynamic systems, Cambridge Scientic Publishers, (2009). 1

[11] A. A. Kilbas, H. M. Srivastava, J. J. Trujillo, Theory and applications of fractional differential equations, Elsevier Science B. V., Amsterdam, (2006). 1

[12] L. Mahto, S. Abbas, A. Favini, Analysis of Caputo impulsive fractional order differential equations with applications, Int. J. Differ. Equ., 2013 (2013), 11 pages. 1

[13] M. W. Michalski, Derivatives of noninteger order and their applications, Dissertationes Math. (Rozprawy Mat.), 328 (1993), 47 pages. 1

[14] K. S. Miller, B. Ross, An introduction to the fractional calculus and differential equations, John Wiley \& Sons, New York, (1993). 1

[15] A. Neamaty, B. Agheli, R. Darzi, Solving Fractional Partial Differential Equation by Using Wavelet Operational Method, J. Math. Comput. Sci., 7 (2013), 230-240. 1

[16] K. B. Oldham, J. Spanier, The Fractional Calculus: Theory and Applications of Differentiation and Integration to Arbitrary Order, Academic Press, New York-London, (1974). 2.2

[17] I. Podlubny, Fractional differential equations, Academic Press, San Diego, (1999). 1

[18] B. M. Randelovic, L. V. Stefanovic, B. M. Dankovic, Numerical solution of impulsive differential equations, Facta Univ. Ser. Math. Inform., 15 (2000), 101-111. 1

[19] S. S. Ray, R. K. Bera, An approximate solution of a nonlinear fractional differential equation by Adomian decomposition method, Appl. Math. Comput., 167 (2005), 561-571.

[20] H. Saeedi, N. Mollahasani, M. M. Moghadam, G. N. Chuev, An operational haarwavelet method for solving fractional volterra integral equations, Int. J. Appl. Math. Comput. Sci., 21 (2011), 535-547. 1

[21] V. E. Tarasov, Fractional dynamics, Springer, Beijing, (2010). 1

[22] J. R. Wang, X. Z. Li, W. Wei, On the natural solution of an impulsive fractional differential equation of order $\mathrm{q} \in(1,2)$, Commun. Nonlinear Sci. Numer. Simul., 17 (2012), 4384-4394. 1

[23] G. T. Wang , L. H. Zhang, G. X. Song, Systems of first order impulsive functional differential equations with deviating arguments and nonlinear boundary conditions, Nonlinear Anal., 74 (2011) ,974-982. 1

[24] Q. Q. Yang, F. W. Liu, I. Turner, Stability and convergence of an effective numerical method for the time-space fractional Fokker-Planck equation with a nonlinear source term, Int. J. Differ. Equ., 2010 (2010), 22 pages. 1

[25] P. Zhuang, F. Liu, V. Anh, I. Turner, Numerical treatment for the fractional Fokker-Planck equation, ANZIAM J., 48 (2006-2007), 759-774. 2.4 